CRYSTALLOGRAPHIC COMMUNICATIONS

ISSN 2056-9890

Received 22 December 2020

Accepted 14 January 2021

Edited by J. T. Mague, Tulane University, USA

Keywords: RuPhos; Buchwald ligand; phosphine; cone angle; crystal structure.

CCDC reference: 2056274

Supporting information: this article has supporting information at journals.iucr.org/e

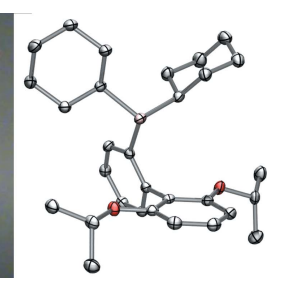

\section{Crystal structure of the RuPhos ligand}

\author{
Kurtis M. Carsch,* William Ho, Kai Hin Lui, Gregory Valtierra, Dilek K. Dogutan,* \\ Daniel G. Nocera and Shao-Liang Zheng*
}

Department of Chemistry and Chemical Biology, Harvard University, 12 Oxford Street, Cambridge, MA, 02138, USA.
*Correspondence e-mail: kcarsch@g.harvard.edu, dkiper@fas.harvard.edu, zheng@chemistry.harvard.edu

Palladium 2-dicyclohexylphosphanyl-2',6'-diisopropoxybiphenyl ( $\mathrm{Pd}-\mathrm{RuPhos}$ ) catalysts demonstrate high catalytic activity for Negishi cross-couplings of sterically hindered aryl halides, for Suzuki-Miyaura cross-couplings of tosylated olefins, and for Buchwald-Hartwig amination of sterically hindered amines. The solid-state structure of the free RuPhos ligand, $\mathrm{C}_{30} \mathrm{H}_{43} \mathrm{O}_{2} \mathrm{P}$, is reported herein for the first time. RuPhos crystallizes in a triclinic cell containing two independent molecules of the phosphine without any lattice solvent. Pertinent bond metrics and comparisons to other phosphine ligands are presented. The structure of RuPhos will be of assistance in the use of this ligand in the design of crosscoupling catalysts.

\section{Chemical context}

Cross-coupling reactions have emerged as a facile method for $\mathrm{C} s p^{2}-\mathrm{Cs} p^{2}$ and $\mathrm{C} s p^{2}-\mathrm{N}$ bond formations. A variety of ancillary phosphine ligands have been observed to mediate challenging Pd-catalyzed cross-coupling reactions (Christmann \& Vilar, 2005). The $\mathrm{Pd}^{0}$ reagent $\mathrm{Pd}_{2}(\mathrm{dba})_{3}(\mathrm{dba}=$ dibenzylideneacetone) in the presence of the ligand 2dicyclohexylphosphanyl-2',6'-diisopropoxybiphenyl (RuPhos, see scheme) is especially effective at catalyzing $\mathrm{Cs} p^{2}-\mathrm{Cs} p^{2}$ bond formation between sterically hindered aryl rings that were previously challenging to couple by traditional crosscoupling methods employing other supporting phosphine ligands (Milne \& Buchwald, 2004). Pd-RuPhos has shown efficacy for a variety of organic substrate transformations, including cross-coupling reactions with sterically hindered aryl halides (Otani et al., 2011; Carsch et al., 2019), stereoselective $\mathrm{Cs} p^{2}-\mathrm{Cs} p^{2}$ bond formation from tosylated olefins ( $\mathrm{Li}$ et al., 2017), Csp $p^{2}-\mathrm{N}$ bond formation afforded by the BuchwaldHartwig amination (Charles et al., 2005), and in the synthesis of new materials, such as the catalyst-transfer polycondensation to furnish polymeric semiconductors such as poly(3alkylthiophenes) (Lee et al., 2020).

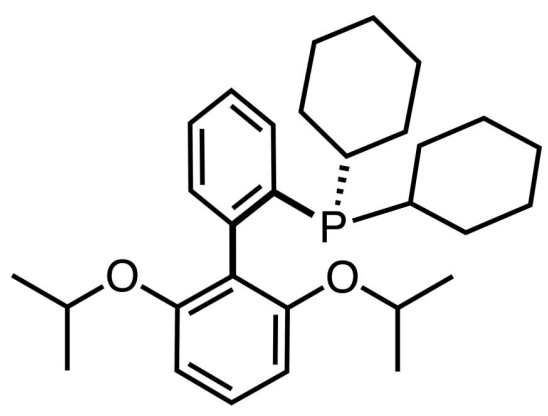


The steric and electronic properties of the ancillary phosphine ligand can have a profound impact on the outcome of the cross-coupling reaction. For example, in the BuchwaldHartwig amination, $\mathrm{Pd}-\mathrm{RuPhos}$ displays high catalytic activity for cross-coupling reactions with sterically hindered substrates such as cyclic secondary amines, whereas the related congener, Pd-BrettPhos, demonstrates high catalytic activity with primary amines (Tian et al., 2020; Charles et al., 2005). The electronic properties and steric profile of the ligand scaffold impact the elementary steps and catalytic performance of the resulting metal complex (van Leeuwen et al., 2000). Recent density functional calculations corroborate the importance of ligand properties on the kinetics of cross-coupling chemistry: the rate-limiting step for $\mathrm{Pd}-\mathrm{RuPhos}$ is predicted to be reductive elimination, while that of the congener Pd-BrettPhos is predicted to be oxidative addition (Tian et al., 2020). Curiously, the solid-state structure of RuPhos remains absent from the literature. Knowledge of the structural metrics of RuPhos will benefit mechanistic and computational studies of this important ligand and will aid in the rational design of new RuPhos-derivative catalysts.

\section{Structural commentary}

The free RuPhos ligand (Fig. 1) was characterized by singlecrystal X-ray diffraction, with pertinent bond metrics listed in Table 1 and experimental structural details delineated in Table 2 . The asymmetric unit contains two independent molecules, RuPhos A and RuPhos B, which differ modestly in conformation. For conciseness, only the structural metrics of RuPhos B are described hereafter, and RuPhos B is simply referred to as RuPhos. Details of the structural metrics of both

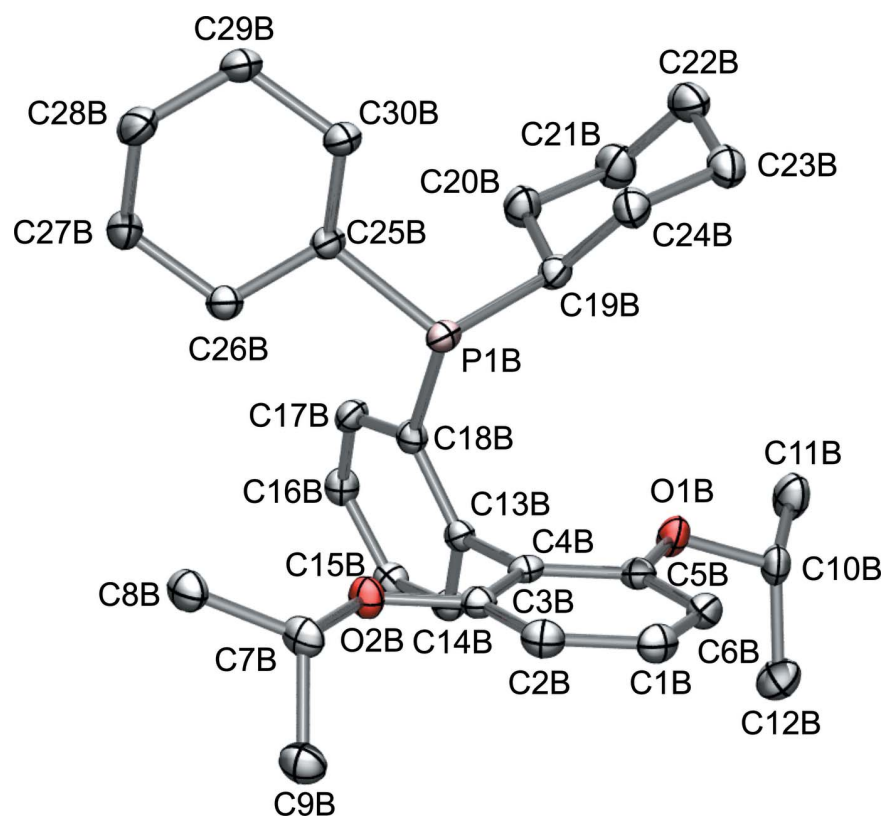

Figure 1

Ellipsoid plot (50\% probability ellipsoids) of RuPhos. Hydrogen atoms are omitted for clarity.
Table 1

Selected geometric parameters $\left(\AA,^{\circ}\right)$ for the two independent molecules RuPhos A and RuPhos B.

Bond distances

\begin{tabular}{lll}
\hline C-C Biaryl & C4-C13 & $1.495(2), 1.499(2)$ \\
Ar-P & C18-P1 & $1.848(2), 1.848(2)$ \\
Cy-P & C19-P1 & $1.876(2), 1.877(2)$ \\
Cy-P & C25-P1 & $1.865(2), 1.862(2)$
\end{tabular}

Selected bond angles

$\mathrm{Ar}-\mathrm{P}-\mathrm{Cy} \quad \mathrm{C} 18-\mathrm{P} 1-\mathrm{C} 25 \quad 101.31(8), 101.86(8)$

$\mathrm{Cy}-\mathrm{P}-\mathrm{Cy} \quad \mathrm{C} 25-\mathrm{P} 1-\mathrm{C} 19$

$\mathrm{Ar}-\mathrm{P}-\mathrm{Cy}$

$\mathrm{C} 18-\mathrm{P} 1-\mathrm{C} 19$

$106.07(8), 105.46(8)$

$98.31(8), 97.03(8)$

Selected torsional angles

Biaryl

Biaryl

$\mathrm{C} 3-\mathrm{C} 4-\mathrm{C} 13-\mathrm{C} 14$

$\mathrm{C} 3-\mathrm{C} 4-\mathrm{C} 13-\mathrm{C} 18$

Biaryl

$\mathrm{C} 5-\mathrm{C} 4-\mathrm{C} 13-\mathrm{C} 14$

$\mathrm{C} 5-\mathrm{C} 4-\mathrm{C} 13-\mathrm{C} 18$

$82.6(2), 73.2(2)$

$97.6(2), 105.8(2)$

$96.1(2), 103.8(2)$

$83.7(2), 77.2(2)$

molecules in the asymmetric unit can be found in the supporting information.

The $\mathrm{C}-\mathrm{C}$ bond lengths (Table S3) in the arene rings differ minimally, ranging from 1.385 (2) to 1.402 (2) $\AA$. The $\mathrm{P}-\mathrm{Cs} p^{2}$ and $\mathrm{P}-\mathrm{Cs} p^{3}$ bond lengths (Table 1) were observed to vary minimally between RuPhos $\mathrm{A}$ and RuPhos $\mathrm{B}$. The $\mathrm{P}-\mathrm{C}_{\mathrm{Ar}}$ bond length ( $\mathrm{P} 1 B-\mathrm{C} 18 B)$ is 1.848 (2) $\AA$ and it is comparable to the previously reported $\mathrm{P}-\mathrm{C}_{\mathrm{Ar}}$ bond lengths in $\mathrm{PPh}_{3}$ (Samouei et al., 2014). As expected, the $\mathrm{P}-\mathrm{C}_{\mathrm{Cy}}$ bond lengths are somewhat longer [P1B-C19B: 1.877 (2) Aं; P1B-C25B: $1.862(2) \AA]$ and comparable to those observed in $\mathrm{PCy}_{3}$ (Davies et al., 1991). The $\mathrm{Cy}(\mathrm{C} 25 B)-\mathrm{P} 1 B-\mathrm{Cy}(19 \mathrm{~B})$ angle is $105.46(8)^{\circ}$. The two $\mathrm{C}_{\mathrm{Ar}}-\mathrm{P}-\mathrm{C}_{\mathrm{Cy}}$ angles are $97.03(8)^{\circ}$ $(\mathrm{C} 18 B-\mathrm{P} 1 B-\mathrm{C} 19 B)$ and $101.86(8)^{\circ}(\mathrm{C} 18 B-\mathrm{P} 1 B-\mathrm{C} 25 B)$. The cyclohexyl rings each adopt a chair conformation relative to $\mathrm{P} 1 B$ and are in an asymmetric orientation relative to the biaryl substituent. No notable interactions between the cyclohexyl rings and other atoms within RuPhos are observed. Additional electron density close to the phosphorus is resolved and assigned to a lone pair rather than a light atom based on its proximity to the phosphorous atom.

The Tolman cone angle quantifies steric and electronic effects of phosphine ligands (Tolman, 1977) and is defined as the angle from a hypothetical metal $M$ located $2.28 \AA$ from the phosphorus atom to the van der Waals radii of the outermost atoms of the phosphine ligand. Half angles are defined by the angle between the $M-\mathrm{P}$ bond and the line between $M-\mathrm{H}_{i}$, where $\mathrm{H}_{i}$ is the outermost atom on the substituent, calculated as:

$\theta_{i}=a_{i}+\sin ^{-1}\left(r_{\mathrm{H}} / d_{i}\right)$

where $\theta_{i}$ is the angle defined between $M-\mathrm{H}_{i}$ and $M-\mathrm{P}$ and $d_{i}$ is the distance between $M$ and $\mathrm{H}_{i}$ (Müller \& Mingos, 1995). For unligated RuPhos, the computed Tolman cone angle is $201.53^{\circ}$ (Table S5). For comparison, the cone angle for $\mathrm{Pd}-$ RuPhos is $198.06^{\circ}$ (Arrechea \& Buchwald, 2016). The RuPhos cone angle is larger than those found in $\mathrm{PCy}_{3}\left(170^{\circ}\right)$ and $\mathrm{PPh}_{3}$ $\left(145^{\circ}\right)$ (Jover \& Cirera, 2019) and is attributed to the steric profile of the biaryl substituent. The cone angle of free RuPhos is larger than the cone angle of Pd-RuPhos, consistent 
with slight modification of the $\mathrm{P}$ hybridization accompanying complexation to the $\mathrm{Pd}$ center.

\section{Supramolecular features}

The crystal packing of RuPhos follows a parallelepiped geometry (Fig. 2), showing two types of intermolecular channel-like interfaces, which alternate in parallel planes. In the first type of interface channel, cyclohexyl substituents from different RuPhos molecules face towards each other. The distance between cyclohexyl rings (Table S6) in different unit cells is less than $4 \AA[d(\mathrm{C} 20 A-\mathrm{C} 22 B)=3.942(3) \AA$, $d(\mathrm{C} 20 A-\mathrm{C} 21 B)=3.977(3) \AA]$, consistent with there being no void in the crystal packing. In the second type of channel, biaryl substituents from different RuPhos molecules arrange themselves in a zigzag offset chain pattern (Fig. S2).

Within the asymmetric unit, RuPhos A and RuPhos B are spaced apart by ca $3 \AA$, as defined by the distance between the

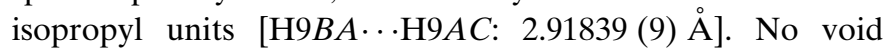
space is observed in the asymmetric unit as evident by a spacefilling model (Fig. S3).

The crystal structure of RuPhos shows consistency in atomic composition and connectivity with the reported structure. Coordination by the phosphine to a metal should occlude equatorial ligands on one side of the metal, though less so than its BrettPhos congener would. The small hindrance of PdRuPhos is thought to contribute to its high catalytic activity for hindered secondary amines while the larger hindrance of BrettPhos contributes to its high catalytic activity for primary amines (Arrechea \& Buchwald, 2016; Tian et al., 2020).

The cone angles of free RuPhos and Pd-RuPhos (Arrechea \& Buchwald, 2016) measure 201.54 and $198.07^{\circ}$, respectively. They are smaller than that of free BrettPhos and Pd-BrettPhos (Dikundwar et al., 2017; DeAngelis et al., 2015), which

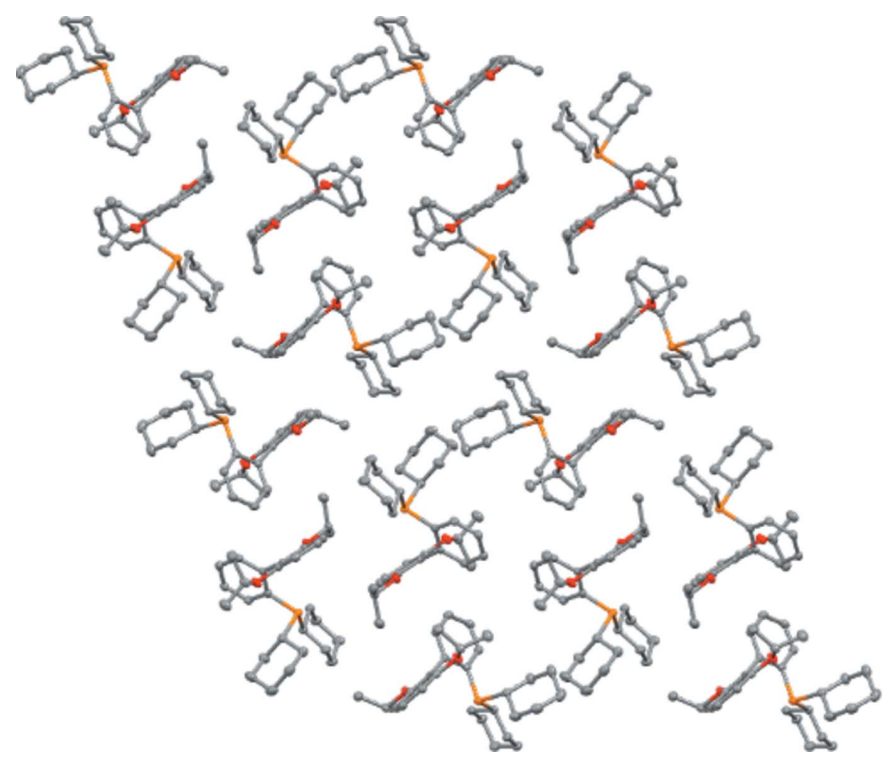

Figure 2

Crystal structure of RuPhos assigned to a parallelepiped geometry, viewed down the $a$ axis (Mercury; Macrae et al., 2020). Color scheme: $\mathrm{P}$ (orange), $\mathrm{C}$ (gray), $\mathrm{O}$ (red).
Table 2

Experimental details.

\begin{tabular}{ll}
\hline Crystal data & \\
Chemical formula & $\mathrm{C}_{30} \mathrm{H}_{43} \mathrm{O}_{2} \mathrm{P}$ \\
$M_{\mathrm{r}}$ & 466.61 \\
Crystal system, space group & Triclinic, $P \overline{1}$ \\
Temperature $(\mathrm{K})$ & 100 \\
$a, b, c(\AA)$ & $9.6160(4), 15.8209(7), 19.0324(9)$ \\
$\alpha, \beta, \gamma\left({ }^{\circ}\right)$ & $71.2052(8), 85.1144(8)$, \\
& $87.9801(9)$ \\
$V\left(\AA^{3}\right)$ & $2731.0(2)$ \\
$Z$ & 4 \\
Radiation type & Mo $K \alpha$ \\
$\mu\left(\mathrm{mm}^{-1}\right)$ & 0.12 \\
Crystal size $(\mathrm{mm})$ & $0.42 \times 0.24 \times 0.12$
\end{tabular}

Data collection

Diffractometer

Absorption correction

$T_{\min }, T_{\max }$

No. of measured, independent and observed $[I>2 \sigma(I)]$ reflections

$R_{\text {int }}$

$(\sin \theta / \lambda)_{\max }\left(\AA^{-1}\right)$

Bruker APEXII CCD
Multi-scan $($ SADABS2016/2;
$\quad$ Krause et al., 2015)
$0.687,0.745$
$55802,9733,7694$

0.044
0.597

$0.042,0.116,1.05$
9733
603
H-atom parameters constrained
$0.49,-0.27$

Refinement

$R\left[F^{2}>2 \sigma\left(F^{2}\right)\right], w R\left(F^{2}\right), S$

No. of reflections

No. of parameters

$\mathrm{H}$-atom treatment

$\Delta \rho_{\max }, \Delta \rho_{\min }\left(\mathrm{e} \AA^{-3}\right)$

$0.49,-0.27$

Computer programs: APEX3 and SAINT (Bruker, 2015), SHELXT2014 (Sheldrick, 2015a), SHELXL2018/3 (Sheldrick, 2015b), SHELXTL (Sheldrick, 2008), and Mercury (Macrae et al., 2020).

are 220.29 and $204.22^{\circ}$, respectively. Because the proportion of $s$ character in the lone pair of a phosphine ligand is inversely proportional to the cone angle of the ligand (Tolman, 1977), the smaller Tolman cone angle of RuPhos implies that RuPhos donates less electron density to its coordinated metal than BrettPhos does. This electronic implication of the RuPhos cone angle corroborates calculations that reductive elimination is the rate-limiting step for $\mathrm{Pd}-\mathrm{RuPhos}$-catalyzed couplings (Tian et al., 2020).

\section{Database survey}

The structure of the unligated RuPhos ligand has not been previously published according to a search of the Cambridge Structural Database using ConQuest 2020.3.0 (CSD, version 5.42, November 2020; Groom et al., 2016). The structure of metallated $\mathrm{Pd}^{\mathrm{II}}$ RuPhos has been reported (Arrechea \& Buchwald, 2016).

\section{Synthesis and crystallization}

RuPhos was purchased from Oakwood Chemical and purified by column chromatography (silica, ethyl acetate). Fractions containing RuPhos were concentrated in vacuo and allowed to stand at room temperature under air with slow evaporation for two weeks in a hexanes/ethyl acetate (10:1) mixture. Colorless plates were observed (Fig. S1) and employed for data collection. 
No evidence for phosphine oxidation was observed in the final refinement. This is attributed to hindered phosphine rotation and the steric profile of the biaryl substituent (Barder et al., 2007).

\section{Refinement}

Crystal data, data collection and structure refinement details are summarized in Table 2 . $\mathrm{H}$ atoms were placed in calculated positions $(\mathrm{C}-\mathrm{H}=0.95-1.00 \AA)$ and refined as riding with $U_{\text {iso }}(\mathrm{H})=1.2 U_{\text {eq }}(\mathrm{C})$ or $1.5 U_{\text {eq }}(\mathrm{C}$-methyl $)$.

\section{Acknowledgements}

We thank N. Ayoub, Rui Sun, and Shelby Elizabeth Elder (Harvard) for helpful discussions.

\section{Funding information}

Funding for this research was provided by Harvard University.

\section{References}

Arrechea, P. L. \& Buchwald, S. L. (2016). J. Am. Chem. Soc. 138, 12486-12493.

Barder, T. E. \& Buchwald, S. L. (2007). J. Am. Chem. Soc. 129, 50965101.

Bruker (2015). APEX3 and SAINT. Bruker AXS Inc., Madison, Wisconsin, USA.

Carsch, K. M., DiMucci, I. M., Iovan, D. A., Li, A., Zheng, S. L., Titus, C. J., Lee, S. J., Irwin, K. D., Nordlund, D., Lancaster, K. M. \& Betley, T. A. (2019). Science, 365, 1138-1143.

Charles, M. D., Schultz, P. \& Buchwald, S. L. (2005). Org. Lett. 7, 3965-3968.
Christmann, U. \& Vilar, R. (2005). Angew. Chem. Int. Ed. 44, 366374.

Davies, J. A., Dutremez, S. \& Pinkerton, A. A. (1991). Inorg. Chem. 30, 2380-2387.

DeAngelis, A. J., Gildner, P. G., Chow, R. \& Colacot, T. J. (2015). J. Org. Chem. 80, 6794-6813.

Dikundwar, A. G., Chodon, P., Thomas, S. P. \& Bhutani, H. (2017). Cryst. Growth Des. 17, 1982-1990.

Groom, C. R., Bruno, I. J., Lightfoot, M. P. \& Ward, S. C. (2016). Acta Cryst. B72, 171-179.

Jover, J. \& Cirera, J. (2019). Dalton Trans. 48, 15036-15048.

Krause, L., Herbst-Irmer, R., Sheldrick, G. M. \& Stalke, D. (2015). J. Appl. Cryst. 48, 3-10.

Lee, J., Park, H., Hwang, S.-H., Lee, I.-H. \& Choi, T.-L. (2020). Macromolecules, 53, 3306-3314.

Leeuwen, P. W. N. M. van, Kamer, P. C. J., Reek, J. N. H. \& Dierkes, P. (2000). Chem. Rev. 100, 2741-2770.

Li, B. X., Le, D. N., Mack, K. A., McClory, A., Lim, N.-K., Cravillion, T., Savage, S., Han, C., Collum, D. B., Zhang, H. \& Gosselin, F. (2017). J. Am. Chem. Soc. 139, 10777-10783.

Macrae, C. F., Sovago, I., Cottrell, S. J., Galek, P. T. A., McCabe, P., Pidcock, E., Platings, M., Shields, G. P., Stevens, J. S., Towler, M. \& Wood, P. A. (2020). J. Appl. Cryst. 53, 226-235.

Milne, J. A. \& Buchwald, S. L. (2004). J. Am. Chem. Soc. 126, 1302813032.

Müller, T. E. \& Mingos, D. M. P. (1995). Transition Met. Chem. 20, 533-539.

Otani, T., Hachiya, M., Hashizume, D., Matsuo, T. \& Tamao, K. (2011). Chem. Asian J. 6, 350-354.

Samouei, H., Miloserdov, F. M., Escudero-Adán, E. C. \& Grushin, V. V. (2014). Organometallics, 33, 7279-7283.

Sheldrick, G. M. (2008). Acta Cryst. A64, 112-122.

Sheldrick, G. M. (2015a). Acta Cryst. C71, 3-8.

Sheldrick, G. M. (2015b). Acta Cryst. A71, 3-8.

Tian, J., Wang, G., Qi, Z. H. \& Ma, J. (2020). ACS Omega, 5, $21385-$ 21391.

Tolman, C. A. (1977). Chem. Rev. 77, 313-348. 


\section{supporting information}

Acta Cryst. (2021). E77, 171-174 [https://doi.org/10.1107/S2056989021000542]

\section{Crystal structure of the RuPhos ligand}

Kurtis M. Carsch, William Ho, Kai Hin Lui, Gregory Valtierra, Dilek K. Dogutan, Daniel G. Nocera and Shao-Liang Zheng

\section{Computing details}

Data collection: APEX3 (Bruker, 2015); cell refinement: SAINT (Bruker, 2015); data reduction: SAINT (Bruker, 2015); program(s) used to solve structure: SHELXT2014 (Sheldrick, 2015a); program(s) used to refine structure: SHELXL2018/3 (Sheldrick, 2015b); molecular graphics: SHELXTL (Sheldrick, 2008); Mercury (Macrae et al., 2020); software used to prepare material for publication: SHELXTL (Sheldrick, 2008).

2-Dicyclohexylphosphanyl-2',6'-bis(propan-2-yloxy)biphenyl

Crystal data

$\mathrm{C}_{30} \mathrm{H}_{43} \mathrm{O}_{2} \mathrm{P}$

$M_{r}=466.61$

Triclinic, $P \overline{1}$

$a=9.6160(4) \AA$

$b=15.8209$ (7) $\AA$

$c=19.0324(9) \AA$

$\alpha=71.2052(8)^{\circ}$

$\beta=85.1144(8)^{\circ}$

$\gamma=87.9801(9)^{\circ}$

$V=2731.0(2) \AA^{3}$

Data collection

Bruker APEXII CCD

diffractometer

Radiation source: fine-focus sealed tube

$\omega$ and phi scans

Absorption correction: multi-scan

(SADABS2016/2; Krause et al., 2015)

$T_{\min }=0.687, T_{\max }=0.745$

55802 measured reflections

\section{Refinement}

Refinement on $F^{2}$

Least-squares matrix: full

$R\left[F^{2}>2 \sigma\left(F^{2}\right)\right]=0.042$

$w R\left(F^{2}\right)=0.116$

$S=1.05$

9733 reflections

603 parameters

0 restraints

Primary atom site location: dual
$Z=4$

$F(000)=1016$

$D_{\mathrm{x}}=1.135 \mathrm{Mg} \mathrm{m}^{-3}$

Mo $K \alpha$ radiation, $\lambda=0.71073 \AA$

Cell parameters from 9987 reflections

$\theta=2.2-24.8^{\circ}$

$\mu=0.12 \mathrm{~mm}^{-1}$

$T=100 \mathrm{~K}$

Plate, colorless

$0.42 \times 0.24 \times 0.12 \mathrm{~mm}$

9733 independent reflections

7694 reflections with $I>2 \sigma(I)$

$R_{\text {int }}=0.044$

$\theta_{\max }=25.1^{\circ}, \theta_{\min }=1.4^{\circ}$

$h=-11 \rightarrow 11$

$k=-18 \rightarrow 18$

$l=-22 \rightarrow 22$

Secondary atom site location: difference Fourier map

Hydrogen site location: inferred from neighbouring sites

$\mathrm{H}$-atom parameters constrained

$w=1 /\left[\sigma^{2}\left(F_{\mathrm{o}}^{2}\right)+(0.0678 P)^{2}+0.4429 P\right]$

where $P=\left(F_{\mathrm{o}}^{2}+2 F_{\mathrm{c}}^{2}\right) / 3$

$(\Delta / \sigma)_{\max }=0.001$

$\Delta \rho_{\max }=0.49 \mathrm{e} \AA^{-3}$

$\Delta \rho_{\min }=-0.27$ e $\AA^{-3}$ 


\section{Special details}

Geometry. All esds (except the esd in the dihedral angle between two 1.s. planes) are estimated using the full covariance matrix. The cell esds are taken into account individually in the estimation of esds in distances, angles and torsion angles; correlations between esds in cell parameters are only used when they are defined by crystal symmetry. An approximate (isotropic) treatment of cell esds is used for estimating esds involving l.s. planes.

Refinement. No significant disordering was present.

Fractional atomic coordinates and isotropic or equivalent isotropic displacement parameters $\left(\AA^{2}\right)$

\begin{tabular}{|c|c|c|c|c|}
\hline & $x$ & $y$ & $z$ & $U_{\text {iso }} * / U_{\text {eq }}$ \\
\hline $\mathrm{P} 1 \mathrm{~A}$ & $0.44121(4)$ & $0.24874(3)$ & $0.10395(2)$ & $0.02025(12)$ \\
\hline O1A & $0.73773(12)$ & $0.26833(8)$ & $0.22546(7)$ & $0.0254(3)$ \\
\hline $\mathrm{O} 2 \mathrm{~A}$ & $0.38076(12)$ & $0.46762(8)$ & $0.12987(7)$ & $0.0260(3)$ \\
\hline $\mathrm{C} 1 \mathrm{~A}$ & $0.76140(18)$ & $0.49580(12)$ & $0.09137(10)$ & $0.0253(4)$ \\
\hline H1A & 0.828797 & 0.538226 & 0.062727 & $0.030^{*}$ \\
\hline $\mathrm{C} 2 \mathrm{~A}$ & $0.80634(18)$ & $0.41479(12)$ & $0.13848(9)$ & $0.0232(4)$ \\
\hline $\mathrm{H} 2 \mathrm{~A}$ & 0.903169 & 0.401717 & 0.142059 & $0.028^{*}$ \\
\hline $\mathrm{C} 3 \mathrm{~A}$ & $0.70763(17)$ & $0.35312(11)$ & $0.18034(9)$ & $0.0205(4)$ \\
\hline $\mathrm{C} 4 \mathrm{~A}$ & $0.56474(17)$ & $0.37326(11)$ & $0.17716(9)$ & $0.0201(4)$ \\
\hline $\mathrm{C} 5 \mathrm{~A}$ & $0.52256(17)$ & $0.45552(11)$ & $0.12887(9)$ & $0.0212(4)$ \\
\hline C6A & $0.62045(18)$ & $0.51673(12)$ & $0.08484(9)$ & $0.0238(4)$ \\
\hline H6A & 0.591592 & 0.571762 & 0.050958 & $0.029^{*}$ \\
\hline C7A & $0.87460(17)$ & $0.24929(12)$ & $0.25305(9)$ & $0.0226(4)$ \\
\hline H7A & 0.947689 & 0.268124 & 0.210533 & $0.027 *$ \\
\hline $\mathrm{C} 8 \mathrm{~A}$ & $0.8784(2)$ & $0.14893(12)$ & $0.28799(11)$ & $0.0327(4)$ \\
\hline H8AA & 0.803622 & 0.130433 & 0.327973 & $0.049^{*}$ \\
\hline H8AB & 0.968856 & 0.130520 & 0.308542 & $0.049^{*}$ \\
\hline H8AC & 0.865337 & 0.120769 & 0.250117 & $0.049^{*}$ \\
\hline C9A & $0.8942(2)$ & $0.29824(13)$ & $0.30753(11)$ & $0.0337(5)$ \\
\hline H9AA & 0.881824 & 0.362507 & 0.283121 & $0.051 *$ \\
\hline H9AB & 0.988436 & 0.286470 & 0.324639 & $0.051^{*}$ \\
\hline H9AC & 0.825244 & 0.277568 & 0.350252 & $0.051^{*}$ \\
\hline $\mathrm{C} 10 \mathrm{~A}$ & $0.32140(18)$ & $0.55063(11)$ & $0.08509(10)$ & $0.0234(4)$ \\
\hline $\mathrm{H} 10 \mathrm{~A}$ & 0.364353 & 0.567120 & 0.032781 & $0.028^{*}$ \\
\hline C11A & $0.16772(18)$ & $0.53064(12)$ & $0.08720(10)$ & $0.0286(4)$ \\
\hline H11A & 0.157521 & 0.479873 & 0.069211 & $0.043^{*}$ \\
\hline H11B & 0.120351 & 0.583090 & 0.055317 & $0.043^{*}$ \\
\hline $\mathrm{H} 11 \mathrm{C}$ & 0.125984 & 0.516023 & 0.138452 & $0.043^{*}$ \\
\hline $\mathrm{C} 12 \mathrm{~A}$ & $0.3421(2)$ & $0.62528(12)$ & $0.11709(10)$ & $0.0281(4)$ \\
\hline $\mathrm{H} 12 \mathrm{~A}$ & 0.302150 & 0.607829 & 0.168801 & $0.042 *$ \\
\hline H12B & 0.295424 & 0.679661 & 0.087671 & $0.042 *$ \\
\hline $\mathrm{H} 12 \mathrm{C}$ & 0.442088 & 0.636519 & 0.115453 & $0.042 *$ \\
\hline $\mathrm{C} 13 \mathrm{~A}$ & $0.45947(16)$ & $0.30853(11)$ & $0.22582(9)$ & $0.0189(4)$ \\
\hline $\mathrm{C} 14 \mathrm{~A}$ & $0.42997(18)$ & $0.30783(12)$ & $0.29927(9)$ & $0.0244(4)$ \\
\hline H14A & 0.478409 & 0.347259 & 0.316983 & $0.029^{*}$ \\
\hline $\mathrm{C} 15 \mathrm{~A}$ & $0.33181(18)$ & $0.25092(12)$ & $0.34640(9)$ & $0.0243(4)$ \\
\hline H15A & 0.312327 & 0.251473 & 0.396033 & $0.029^{*}$ \\
\hline C16A & $0.26173(17)$ & $0.19284(12)$ & $0.32077(9)$ & $0.0228(4)$ \\
\hline
\end{tabular}




\begin{tabular}{|c|c|c|c|c|}
\hline $\mathrm{H} 16 \mathrm{~A}$ & 0.193853 & 0.153456 & 0.352835 & $0.027^{*}$ \\
\hline C17A & $0.29094(17)$ & $0.19236(11)$ & $0.24816(9)$ & $0.0219(4)$ \\
\hline H17A & 0.242364 & 0.152374 & 0.231117 & $0.026^{*}$ \\
\hline $\mathrm{C} 18 \mathrm{~A}$ & $0.39021(16)$ & $0.24941(11)$ & $0.19962(9)$ & $0.0191(4)$ \\
\hline C19A & $0.56208(17)$ & $0.14958(11)$ & $0.12748(9)$ & $0.0225(4)$ \\
\hline H19A & 0.623416 & 0.158877 & 0.164187 & $0.027^{*}$ \\
\hline $\mathrm{C} 20 \mathrm{~A}$ & $0.49358(18)$ & $0.05907(12)$ & $0.16617(11)$ & $0.0277(4)$ \\
\hline $\mathrm{H} 20 \mathrm{~A}$ & 0.430588 & 0.046087 & 0.132477 & $0.033^{*}$ \\
\hline $\mathrm{H} 20 \mathrm{~B}$ & 0.436623 & 0.061208 & 0.211426 & $0.033^{*}$ \\
\hline $\mathrm{C} 21 \mathrm{~A}$ & $0.6032(2)$ & $-0.01574(13)$ & $0.18785(12)$ & $0.0336(5)$ \\
\hline $\mathrm{H} 21 \mathrm{~A}$ & 0.660653 & -0.005816 & 0.225178 & $0.040^{*}$ \\
\hline $\mathrm{H} 21 \mathrm{~B}$ & 0.555675 & -0.073817 & 0.210701 & $0.040^{*}$ \\
\hline $\mathrm{C} 22 \mathrm{~A}$ & $0.6969(2)$ & -0.01855 (13) & $0.12017(12)$ & $0.0358(5)$ \\
\hline $\mathrm{H} 22 \mathrm{~A}$ & 0.768857 & -0.065767 & 0.135702 & $0.043 *$ \\
\hline H22B & 0.640685 & -0.033274 & 0.084521 & $0.043 *$ \\
\hline $\mathrm{C} 23 \mathrm{~A}$ & $0.7672(2)$ & $0.07093(14)$ & $0.08252(11)$ & $0.0343(5)$ \\
\hline $\mathrm{H} 23 \mathrm{~A}$ & 0.824561 & 0.068569 & 0.037461 & $0.041^{*}$ \\
\hline $\mathrm{H} 23 \mathrm{~B}$ & 0.830192 & 0.082695 & 0.116769 & $0.041^{*}$ \\
\hline $\mathrm{C} 24 \mathrm{~A}$ & $0.66063(19)$ & $0.14738(14)$ & $0.06047(10)$ & $0.0317(4)$ \\
\hline $\mathrm{H} 24 \mathrm{~A}$ & 0.710452 & 0.204885 & 0.039698 & $0.038^{*}$ \\
\hline $\mathrm{H} 24 \mathrm{~B}$ & 0.605455 & 0.139840 & 0.021323 & $0.038^{*}$ \\
\hline $\mathrm{C} 25 \mathrm{~A}$ & $0.27731(17)$ & $0.20963(11)$ & $0.07905(9)$ & 0.0208 (4) \\
\hline $\mathrm{H} 25 \mathrm{~A}$ & 0.247972 & 0.152025 & 0.117565 & $0.025^{*}$ \\
\hline $\mathrm{C} 26 \mathrm{~A}$ & $0.16401(17)$ & $0.28097(11)$ & $0.07721(9)$ & $0.0228(4)$ \\
\hline $\mathrm{H} 26 \mathrm{~A}$ & 0.147661 & 0.287411 & 0.127304 & $0.027^{*}$ \\
\hline H26B & 0.197014 & 0.339067 & 0.042142 & $0.027^{*}$ \\
\hline $\mathrm{C} 27 \mathrm{~A}$ & $0.02658(18)$ & $0.25724(13)$ & $0.05328(10)$ & $0.0272(4)$ \\
\hline $\mathrm{H} 27 \mathrm{~A}$ & -0.042876 & 0.305446 & 0.051484 & $0.033^{*}$ \\
\hline $\mathrm{H} 27 \mathrm{~B}$ & -0.010478 & 0.201445 & 0.090221 & $0.033^{*}$ \\
\hline C28A & 0.04904 (19) & $0.24489(13)$ & $-0.02343(10)$ & $0.0287(4)$ \\
\hline $\mathrm{H} 28 \mathrm{~A}$ & -0.039501 & 0.226291 & -0.036863 & $0.034^{*}$ \\
\hline $\mathrm{H} 28 \mathrm{~B}$ & 0.077098 & 0.302388 & -0.061213 & $0.034^{*}$ \\
\hline $\mathrm{C} 29 \mathrm{~A}$ & $0.16146(18)$ & $0.17469(12)$ & $-0.02343(10)$ & $0.0265(4)$ \\
\hline H29A & 0.178402 & 0.170659 & -0.074302 & $0.032 *$ \\
\hline H29B & 0.128080 & 0.115811 & 0.010016 & $0.032 *$ \\
\hline $\mathrm{C} 30 \mathrm{~A}$ & $0.29839(18)$ & $0.19636(12)$ & $0.00209(9)$ & $0.0232(4)$ \\
\hline $\mathrm{H} 30 \mathrm{~A}$ & 0.337947 & 0.251408 & -0.034815 & $0.028^{*}$ \\
\hline H30B & 0.366119 & 0.147097 & 0.004464 & $0.028^{*}$ \\
\hline P1B & $1.03926(4)$ & $0.27391(3)$ & $0.64698(2)$ & $0.01980(12)$ \\
\hline O1B & $0.73186(12)$ & $0.17442(8)$ & $0.56160(6)$ & $0.0247(3)$ \\
\hline $\mathrm{O} 2 \mathrm{~B}$ & $1.08772(12)$ & $0.37786(8)$ & $0.45063(6)$ & $0.0236(3)$ \\
\hline $\mathrm{C} 1 \mathrm{~B}$ & $0.70695(18)$ & $0.41179(12)$ & $0.45028(9)$ & $0.0243(4)$ \\
\hline H1B & 0.639525 & 0.457095 & 0.431652 & $0.029^{*}$ \\
\hline $\mathrm{C} 2 \mathrm{~B}$ & $0.66229(18)$ & $0.32608(12)$ & $0.49049(9)$ & $0.0232(4)$ \\
\hline $\mathrm{H} 2 \mathrm{~B}$ & 0.565622 & 0.312511 & 0.499134 & $0.028^{*}$ \\
\hline $\mathrm{C} 3 \mathrm{~B}$ & $0.76187(17)$ & $0.26046(11)$ & $0.51789(9)$ & $0.0198(4)$ \\
\hline $\mathrm{C} 4 \mathrm{~B}$ & $0.90492(17)$ & $0.27906(11)$ & $0.50426(9)$ & $0.0185(4)$ \\
\hline $\mathrm{C} 5 \mathrm{~B}$ & $0.94642(17)$ & $0.36631(11)$ & $0.46270(9)$ & $0.0201(4)$ \\
\hline
\end{tabular}




\begin{tabular}{|c|c|c|c|c|}
\hline C6B & $0.84761(18)$ & $0.43300(12)$ & $0.43657(9)$ & $0.0238(4)$ \\
\hline H6B & 0.876068 & 0.492322 & 0.409687 & $0.029 *$ \\
\hline C7B & $0.59642(17)$ & $0.13792(12)$ & $0.56147(10)$ & $0.0239(4)$ \\
\hline H7B & 0.521795 & 0.179088 & 0.571536 & $0.029 *$ \\
\hline $\mathrm{C} 8 \mathrm{~B}$ & 0.59304 (19) & $0.05138(13)$ & $0.62519(11)$ & $0.0335(5)$ \\
\hline H8BA & 0.667611 & 0.011687 & 0.615704 & $0.050 *$ \\
\hline H8BB & 0.502508 & 0.022705 & 0.629810 & $0.050 *$ \\
\hline $\mathrm{H} 8 \mathrm{BC}$ & 0.606787 & 0.063489 & 0.671467 & $0.050 *$ \\
\hline C9B & $0.5798(2)$ & $0.12544(13)$ & $0.48696(11)$ & $0.0323(4)$ \\
\hline H9BA & 0.589932 & 0.183135 & 0.447410 & $0.049 *$ \\
\hline H9BB & 0.487089 & 0.101298 & 0.487438 & $0.049 *$ \\
\hline H9BC & 0.651476 & 0.083819 & 0.477855 & $0.049 *$ \\
\hline $\mathrm{C} 10 \mathrm{~B}$ & $1.14318(18)$ & $0.46379(11)$ & $0.40493(9)$ & $0.0244(4)$ \\
\hline H10B & 1.093593 & 0.512342 & 0.420388 & $0.029 *$ \\
\hline $\mathrm{C} 11 \mathrm{~B}$ & 1.29504 (19) & $0.46067(13)$ & $0.42091(10)$ & $0.0283(4)$ \\
\hline H11D & 1.301510 & 0.450503 & 0.474117 & $0.042 *$ \\
\hline H11E & 1.339250 & 0.517506 & 0.391998 & $0.042 *$ \\
\hline $\mathrm{H} 11 \mathrm{~F}$ & 1.342733 & 0.411976 & 0.406959 & $0.042 *$ \\
\hline $\mathrm{C} 12 \mathrm{~B}$ & $1.1272(2)$ & $0.47910(13)$ & $0.32287(10)$ & $0.0301(4)$ \\
\hline H12D & 1.178641 & 0.432585 & 0.307474 & $0.045^{*}$ \\
\hline $\mathrm{H} 12 \mathrm{E}$ & 1.164566 & 0.537774 & 0.293236 & $0.045^{*}$ \\
\hline $\mathrm{H} 12 \mathrm{~F}$ & 1.028194 & 0.476797 & 0.315047 & $0.045^{*}$ \\
\hline C13B & $1.01048(16)$ & $0.20602(11)$ & $0.53049(9)$ & $0.0177(3)$ \\
\hline C14B & $1.03032(17)$ & $0.14347(11)$ & $0.49285(9)$ & $0.0215(4)$ \\
\hline H14B & 0.976273 & 0.147910 & 0.452260 & $0.026^{*}$ \\
\hline C15B & $1.12756(17)$ & $0.07502(11)$ & $0.51373(9)$ & $0.0227(4)$ \\
\hline H15B & 1.139771 & 0.032658 & 0.487868 & $0.027 *$ \\
\hline $\mathrm{C} 16 \mathrm{~B}$ & $1.20710(17)$ & $0.06889(11)$ & $0.57289(9)$ & $0.0224(4)$ \\
\hline H16B & 1.275321 & 0.022894 & 0.587028 & $0.027 *$ \\
\hline C17B & $1.18669(17)$ & $0.13003(11)$ & $0.61122(9)$ & $0.0217(4)$ \\
\hline H17B & 1.241577 & 0.125313 & 0.651551 & $0.026^{*}$ \\
\hline C18B & $1.08682(17)$ & $0.19868(11)$ & $0.59176(9)$ & $0.0194(4)$ \\
\hline C19B & $0.91541(17)$ & $0.19627(11)$ & $0.71832(9)$ & $0.0223(4)$ \\
\hline H19B & 0.856780 & 0.169966 & 0.689800 & $0.027 *$ \\
\hline $\mathrm{C} 20 \mathrm{~B}$ & $0.98146(18)$ & $0.11693(12)$ & $0.77526(10)$ & $0.0286(4)$ \\
\hline $\mathrm{H} 20 \mathrm{C}$ & 1.041014 & 0.082756 & 0.748730 & $0.034^{*}$ \\
\hline H20D & 1.041788 & 0.139264 & 0.804967 & $0.034^{*}$ \\
\hline $\mathrm{C} 21 \mathrm{~B}$ & $0.8713(2)$ & $0.05504(13)$ & $0.82749(11)$ & $0.0345(5)$ \\
\hline $\mathrm{H} 21 \mathrm{C}$ & 0.817380 & 0.027502 & 0.798613 & $0.041 *$ \\
\hline H21D & 0.918013 & 0.006570 & 0.865178 & $0.041^{*}$ \\
\hline $\mathrm{C} 22 \mathrm{~B}$ & $0.7725(2)$ & $0.10601(14)$ & $0.86646(11)$ & $0.0353(5)$ \\
\hline $\mathrm{H} 22 \mathrm{C}$ & 0.699449 & 0.065258 & 0.897991 & $0.042 *$ \\
\hline H22D & 0.824911 & 0.128745 & 0.899127 & $0.042 *$ \\
\hline $\mathrm{C} 23 \mathrm{~B}$ & 0.70455 (19) & $0.18366(13)$ & $0.80992(11)$ & $0.0320(4)$ \\
\hline $\mathrm{H} 23 \mathrm{C}$ & 0.643527 & 0.217229 & 0.836379 & $0.038^{*}$ \\
\hline $\mathrm{H} 23 \mathrm{D}$ & 0.645504 & 0.160359 & 0.780269 & $0.038^{*}$ \\
\hline C24B & $0.81342(18)$ & $0.24663(12)$ & $0.75773(10)$ & $0.0272(4)$ \\
\hline $\mathrm{H} 24 \mathrm{C}$ & 0.866091 & 0.274836 & 0.786643 & $0.033 *$ \\
\hline
\end{tabular}




$\begin{array}{lllll}\text { H24D } & 0.765810 & 0.294559 & 0.720036 & 0.033^{*} \\ \text { C25B } & 1.20122(17) & 0.27172(11) & 0.69469(9) & 0.0208(4) \\ \text { H25B } & 1.228156 & 0.208342 & 0.720520 & 0.025^{*} \\ \text { C26B } & 1.31797(17) & 0.31641(12) & 0.63462(9) & 0.0222(4) \\ \text { H26C } & 1.334558 & 0.281686 & 0.599700 & 0.027^{*} \\ \text { H26D } & 1.287521 & 0.377284 & 0.605919 & 0.027^{*} \\ \text { C27B } & 1.45393(18) & 0.32211(13) & 0.66890(10) & 0.0282(4) \\ \text { H27C } & 1.524993 & 0.353401 & 0.629089 & 0.034^{*} \\ \text { H27D } & 1.489293 & 0.261125 & 0.693694 & 0.034^{*} \\ \text { C28B } & 1.4312(2) & 0.37213(14) & 0.72561(10) & 0.0321(4) \\ \text { H28C } & 1.519260 & 0.372367 & 0.748948 & 0.039^{*} \\ \text { H28D } & 1.404605 & 0.434862 & 0.699914 & 0.039^{*} \\ \text { C29B } & 1.31674(19) & 0.32821(13) & 0.78577(10) & 0.0289(4) \\ \text { H29C } & 1.300406 & 0.363504 & 0.820269 & 0.035^{*} \\ \text { H29D } & 1.347692 & 0.267590 & 0.814789 & 0.035^{*} \\ \text { C30B } & 1.18020(18) & 0.32175(12) & 0.75184(9) & 0.0245(4) \\ \text { H30C } & 1.144103 & 0.382614 & 0.727317 & 0.029^{*} \\ \text { H30D } & 1.109851 & 0.290248 & 0.791983 & 0.029^{*}\end{array}$

Atomic displacement parameters $\left(\AA^{2}\right)$

\begin{tabular}{lllllll}
\hline & $U^{11}$ & $U^{22}$ & $U^{33}$ & $U^{12}$ & $U^{13}$ & $U^{23}$ \\
\hline P1A & $0.0213(2)$ & $0.0221(2)$ & $0.0174(2)$ & $-0.00280(18)$ & $-0.00069(17)$ & $-0.00635(18)$ \\
O1A & $0.0190(6)$ & $0.0217(7)$ & $0.0317(7)$ & $-0.0014(5)$ & $-0.0075(5)$ & $-0.0017(5)$ \\
O2A & $0.0212(6)$ & $0.0212(7)$ & $0.0308(7)$ & $0.0014(5)$ & $-0.0038(5)$ & $-0.0016(5)$ \\
C1A & $0.0266(9)$ & $0.0263(10)$ & $0.0233(9)$ & $-0.0079(8)$ & $0.0009(7)$ & $-0.0082(8)$ \\
C2A & $0.0209(9)$ & $0.0256(10)$ & $0.0242(9)$ & $-0.0019(7)$ & $-0.0023(7)$ & $-0.0092(8)$ \\
C3A & $0.0228(9)$ & $0.0212(9)$ & $0.0187(8)$ & $-0.0007(7)$ & $-0.0051(7)$ & $-0.0070(7)$ \\
C4A & $0.0230(9)$ & $0.0201(9)$ & $0.0188(8)$ & $-0.0012(7)$ & $-0.0026(7)$ & $-0.0081(7)$ \\
C5A & $0.0212(9)$ & $0.0226(9)$ & $0.0214(9)$ & $-0.0003(7)$ & $-0.0021(7)$ & $-0.0092(7)$ \\
C6A & $0.0287(10)$ & $0.0217(9)$ & $0.0206(9)$ & $-0.0019(7)$ & $-0.0018(7)$ & $-0.0058(7)$ \\
C7A & $0.0172(8)$ & $0.0266(10)$ & $0.0229(9)$ & $0.0015(7)$ & $-0.0047(7)$ & $-0.0059(8)$ \\
C8A & $0.0289(10)$ & $0.0289(11)$ & $0.0387(11)$ & $0.0028(8)$ & $-0.0109(8)$ & $-0.0068(9)$ \\
C9A & $0.0408(11)$ & $0.0334(11)$ & $0.0284(10)$ & $0.0036(9)$ & $-0.0133(9)$ & $-0.0099(9)$ \\
C10A & $0.0273(9)$ & $0.0200(9)$ & $0.0209(9)$ & $0.0036(7)$ & $-0.0048(7)$ & $-0.0035(7)$ \\
C11A & $0.0271(10)$ & $0.0286(10)$ & $0.0315(10)$ & $0.0048(8)$ & $-0.0072(8)$ & $-0.0109(8)$ \\
C12A & $0.0328(10)$ & $0.0269(10)$ & $0.0256(10)$ & $0.0008(8)$ & $-0.0022(8)$ & $-0.0099(8)$ \\
C13A & $0.0183(8)$ & $0.0171(9)$ & $0.0202(8)$ & $0.0036(7)$ & $-0.0040(7)$ & $-0.0043(7)$ \\
C14A & $0.0260(9)$ & $0.0259(10)$ & $0.0235(9)$ & $-0.0003(7)$ & $-0.0045(7)$ & $-0.0103(8)$ \\
C15A & $0.0264(9)$ & $0.0293(10)$ & $0.0173(9)$ & $0.0024(8)$ & $-0.0030(7)$ & $-0.0076(8)$ \\
C16A & $0.0206(9)$ & $0.0240(9)$ & $0.0200(9)$ & $-0.0010(7)$ & $0.0011(7)$ & $-0.0025(7)$ \\
C17A & $0.0212(9)$ & $0.0223(9)$ & $0.0228(9)$ & $-0.0019(7)$ & $-0.0037(7)$ & $-0.0075(7)$ \\
C18A & $0.0192(8)$ & $0.0204(9)$ & $0.0171(8)$ & $0.0029(7)$ & $-0.0031(7)$ & $-0.0049(7)$ \\
C19A & $0.0211(9)$ & $0.0258(10)$ & $0.0217(9)$ & $0.0004(7)$ & $-0.0033(7)$ & $-0.0089(7)$ \\
C20A & $0.0243(9)$ & $0.0233(10)$ & $0.0349(10)$ & $-0.0005(7)$ & $-0.0029(8)$ & $-0.0085(8)$ \\
C21A & $0.0304(10)$ & $0.0241(10)$ & $0.0454(12)$ & $0.0001(8)$ & $-0.0067(9)$ & $-0.0091(9)$ \\
C22A & $0.0331(11)$ & $0.0382(12)$ & $0.0455(12)$ & $0.0123(9)$ & $-0.0159(9)$ & $-0.0246(10)$ \\
C23A & $0.0276(10)$ & $0.0470(13)$ & $0.0282(10)$ & $0.0096(9)$ & $-0.0017(8)$ & $-0.0130(9)$ \\
& & & & & &
\end{tabular}




\begin{tabular}{|c|c|c|c|c|c|c|}
\hline $\mathrm{C} 24 \mathrm{~A}$ & $0.0287(10)$ & $0.0406(12)$ & $0.0240(10)$ & $0.0065(9)$ & $-0.0001(8)$ & $-0.0092(9)$ \\
\hline $\mathrm{C} 25 \mathrm{~A}$ & $0.0229(9)$ & $0.0207(9)$ & $0.0193(9)$ & $-0.0012(7)$ & $-0.0018(7)$ & $-0.0068(7)$ \\
\hline $\mathrm{C} 26 \mathrm{~A}$ & $0.0263(9)$ & $0.0224(9)$ & $0.0197(9)$ & $0.0015(7)$ & $-0.0021(7)$ & $-0.0070(7)$ \\
\hline C27A & $0.0252(9)$ & $0.0332(11)$ & $0.0260(10)$ & $0.0055(8)$ & $-0.0063(7)$ & $-0.0127(8)$ \\
\hline $\mathrm{C} 28 \mathrm{~A}$ & $0.0260(10)$ & $0.0339(11)$ & $0.0284(10)$ & $0.0035(8)$ & $-0.0081(8)$ & $-0.0122(8)$ \\
\hline C29A & $0.0299(10)$ & $0.0300(10)$ & $0.0231(9)$ & $0.0006(8)$ & $-0.0053(7)$ & $-0.0127(8)$ \\
\hline $\mathrm{C} 30 \mathrm{~A}$ & $0.0245(9)$ & $0.0255(10)$ & $0.0224(9)$ & $0.0020(7)$ & $-0.0047(7)$ & $-0.0110(8)$ \\
\hline P1B & $0.0204(2)$ & $0.0215(2)$ & $0.0189(2)$ & $0.00027(18)$ & $-0.00450(17)$ & $-0.00770(18)$ \\
\hline O1B & $0.0187(6)$ & $0.0236(7)$ & $0.0270(7)$ & $-0.0037(5)$ & $-0.0050(5)$ & $-0.0001(5)$ \\
\hline $\mathrm{O} 2 \mathrm{~B}$ & $0.0223(6)$ & $0.0189(6)$ & $0.0257(6)$ & $-0.0036(5)$ & $0.0004(5)$ & $-0.0021(5)$ \\
\hline $\mathrm{C} 1 \mathrm{~B}$ & $0.0262(9)$ & $0.0231(10)$ & $0.0243(9)$ & $0.0051(7)$ & $-0.0043(7)$ & $-0.0083(8)$ \\
\hline $\mathrm{C} 2 \mathrm{~B}$ & $0.0205(9)$ & $0.0282(10)$ & $0.0216(9)$ & $0.0009(7)$ & $-0.0032(7)$ & $-0.0086(8)$ \\
\hline $\mathrm{C} 3 \mathrm{~B}$ & $0.0234(9)$ & $0.0197(9)$ & $0.0166(8)$ & $-0.0019(7)$ & $-0.0022(7)$ & $-0.0057(7)$ \\
\hline C4B & $0.0216(9)$ & $0.0205(9)$ & $0.0152(8)$ & $0.0001(7)$ & $-0.0037(6)$ & $-0.0078(7)$ \\
\hline C5B & $0.0226(9)$ & $0.0218(9)$ & $0.0173(8)$ & $-0.0015(7)$ & $-0.0019(7)$ & $-0.0078(7)$ \\
\hline C6B & $0.0295(10)$ & $0.0184(9)$ & $0.0225(9)$ & $-0.0001(7)$ & $-0.0023(7)$ & $-0.0049(7)$ \\
\hline C7B & $0.0153(8)$ & $0.0259(10)$ & $0.0280(9)$ & $-0.0026(7)$ & $-0.0007(7)$ & $-0.0049(8)$ \\
\hline C8B & $0.0259(10)$ & $0.0323(11)$ & $0.0360(11)$ & $-0.0073(8)$ & $-0.0033(8)$ & $-0.0011(9)$ \\
\hline C9B & $0.0337(11)$ & $0.0306(11)$ & $0.0344(11)$ & $-0.0029(8)$ & $-0.0074(8)$ & $-0.0114(9)$ \\
\hline C10B & $0.0308(10)$ & $0.0171(9)$ & $0.0234(9)$ & $-0.0063(7)$ & $0.0022(7)$ & $-0.0044(7)$ \\
\hline C11B & $0.0302(10)$ & $0.0286(10)$ & $0.0269(10)$ & $-0.0099(8)$ & $0.0027(8)$ & $-0.0101(8)$ \\
\hline C12B & $0.0358(11)$ & $0.0266(10)$ & $0.0250(10)$ & $-0.0033(8)$ & $0.0004(8)$ & $-0.0048(8)$ \\
\hline C13B & $0.0183(8)$ & $0.0167(9)$ & $0.0167(8)$ & $-0.0039(7)$ & $-0.0002(6)$ & $-0.0032(7)$ \\
\hline C14B & $0.0224(9)$ & $0.0241(9)$ & $0.0177(8)$ & $-0.0049(7)$ & $-0.0016(7)$ & $-0.0059(7)$ \\
\hline C15B & $0.0250(9)$ & $0.0202(9)$ & $0.0236(9)$ & $-0.0025(7)$ & $0.0007(7)$ & $-0.0086(7)$ \\
\hline C16B & $0.0212(9)$ & $0.0184(9)$ & $0.0255(9)$ & $0.0003(7)$ & $-0.0018(7)$ & $-0.0041(7)$ \\
\hline C17B & $0.0198(9)$ & $0.0239(9)$ & $0.0208(9)$ & $-0.0013(7)$ & $-0.0058(7)$ & $-0.0053(7)$ \\
\hline C18B & $0.0186(8)$ & $0.0195(9)$ & $0.0199(8)$ & $-0.0033(7)$ & $0.0003(7)$ & $-0.0061(7)$ \\
\hline C19B & $0.0202(9)$ & $0.0247(9)$ & $0.0242(9)$ & $-0.0017(7)$ & $-0.0044(7)$ & $-0.0098(8)$ \\
\hline C20B & $0.0245(9)$ & $0.0296(10)$ & $0.0278(10)$ & $-0.0016(8)$ & $-0.0026(8)$ & $-0.0036(8)$ \\
\hline $\mathrm{C} 21 \mathrm{~B}$ & $0.0325(11)$ & $0.0329(11)$ & $0.0313(11)$ & $-0.0061(9)$ & -0.0015 & $-0.0003(9)$ \\
\hline $\mathrm{C} 22 \mathrm{~B}$ & $0.0346(11)$ & $0.0451(13)$ & $0.0270(10)$ & $-0.0155(9)$ & $0.0035(8)$ & $-0.0124(9)$ \\
\hline C23B & $0.0267(10)$ & $0.0392(12)$ & $0.0353(11)$ & $-0.0069(8)$ & $0.0036(8)$ & $-0.0200(9)$ \\
\hline C24B & $0.0231(9)$ & $0.0311(10)$ & $0.0300(10)$ & $-0.0011(8)$ & $0.0000(8)$ & $-0.0138(8)$ \\
\hline $\mathrm{C} 25 \mathrm{~B}$ & $0.0212(9)$ & $0.0224(9)$ & $0.0194(9)$ & $-0.0008(7)$ & $-0.0036(7)$ & $-0.0069(7)$ \\
\hline C26B & $0.0241(9)$ & $0.0236(9)$ & $0.0191(9)$ & $-0.0035(7)$ & $-0.0017(7)$ & $-0.0067(7)$ \\
\hline C27B & $0.0232(9)$ & $0.0362(11)$ & $0.0243(9)$ & $-0.0073(8)$ & $-0.0010(7)$ & $-0.0081(8)$ \\
\hline $\mathrm{C} 28 \mathrm{~B}$ & $0.0306(10)$ & $0.0409(12)$ & $0.0262(10)$ & $-0.0132(9)$ & -0.0050 & -0.0105 (9) \\
\hline C29B & $0.0308(10)$ & $0.0378(11)$ & $0.0199(9)$ & $-0.0059(8)$ & $-0.0041(8)$ & $-0.0108(8)$ \\
\hline $\mathrm{C} 30 \mathrm{~B}$ & $0.0251(9)$ & $0.0303(10)$ & $0.0201(9)$ & $-0.0047(8)$ & $-0.0026(7)$ & $-0.0102(8)$ \\
\hline
\end{tabular}

Geometric parameters $\left(\AA,{ }^{\circ}\right)$

\begin{tabular}{llll}
\hline P1A-C18A & $1.8482(16)$ & P1B-C18B & $1.8482(17)$ \\
P1A-C25A & $1.8645(17)$ & P1B-C25B & $1.8624(17)$ \\
P1A-C19A & $1.8762(17)$ & P1B-C19B & $1.8771(17)$ \\
O1A-C3A & $1.376(2)$ & O1B-C3B & $1.373(2)$ \\
O1A-C7A & $1.4444(19)$ & O1B-C7B & $1.4437(19)$
\end{tabular}




\begin{tabular}{|c|c|c|c|}
\hline $\mathrm{O} 2 \mathrm{~A}-\mathrm{C} 5 \mathrm{~A}$ & $1.370(2)$ & $\mathrm{O} 2 \mathrm{~B}-\mathrm{C} 5 \mathrm{~B}$ & $1.367(2)$ \\
\hline $\mathrm{O} 2 \mathrm{~A}-\mathrm{C} 10 \mathrm{~A}$ & $1.444(2)$ & $\mathrm{O} 2 \mathrm{~B}-\mathrm{C} 10 \mathrm{~B}$ & $1.449(2)$ \\
\hline $\mathrm{C} 1 \mathrm{~A}-\mathrm{C} 2 \mathrm{~A}$ & $1.386(3)$ & $\mathrm{C} 1 \mathrm{~B}-\mathrm{C} 2 \mathrm{~B}$ & $1.388(2)$ \\
\hline $\mathrm{C} 1 \mathrm{~A}-\mathrm{C} 6 \mathrm{~A}$ & $1.390(2)$ & $\mathrm{C} 1 \mathrm{~B}-\mathrm{C} 6 \mathrm{~B}$ & $1.388(2)$ \\
\hline $\mathrm{C} 1 \mathrm{~A}-\mathrm{H} 1 \mathrm{~A}$ & 0.9500 & $\mathrm{C} 1 \mathrm{~B}-\mathrm{H} 1 \mathrm{~B}$ & 0.9500 \\
\hline $\mathrm{C} 2 \mathrm{~A}-\mathrm{C} 3 \mathrm{~A}$ & $1.387(2)$ & $\mathrm{C} 2 \mathrm{~B}-\mathrm{C} 3 \mathrm{~B}$ & $1.390(2)$ \\
\hline $\mathrm{C} 2 \mathrm{~A}-\mathrm{H} 2 \mathrm{~A}$ & 0.9500 & $\mathrm{C} 2 \mathrm{~B}-\mathrm{H} 2 \mathrm{~B}$ & 0.9500 \\
\hline $\mathrm{C} 3 \mathrm{~A}-\mathrm{C} 4 \mathrm{~A}$ & $1.401(2)$ & $\mathrm{C} 3 \mathrm{~B}-\mathrm{C} 4 \mathrm{~B}$ & $1.402(2)$ \\
\hline $\mathrm{C} 4 \mathrm{~A}-\mathrm{C} 5 \mathrm{~A}$ & $1.399(2)$ & $\mathrm{C} 4 \mathrm{~B}-\mathrm{C} 5 \mathrm{~B}$ & $1.404(2)$ \\
\hline $\mathrm{C} 4 \mathrm{~A}-\mathrm{C} 13 \mathrm{~A}$ & $1.495(2)$ & $\mathrm{C} 4 \mathrm{~B}-\mathrm{C} 13 \mathrm{~B}$ & $1.499(2)$ \\
\hline $\mathrm{C} 5 \mathrm{~A}-\mathrm{C} 6 \mathrm{~A}$ & $1.390(2)$ & $\mathrm{C} 5 \mathrm{~B}-\mathrm{C} 6 \mathrm{~B}$ & $1.391(2)$ \\
\hline $\mathrm{C} 6 \mathrm{~A}-\mathrm{H} 6 \mathrm{~A}$ & 0.9500 & $\mathrm{C} 6 \mathrm{~B}-\mathrm{H} 6 \mathrm{~B}$ & 0.9500 \\
\hline $\mathrm{C} 7 \mathrm{~A}-\mathrm{C} 9 \mathrm{~A}$ & $1.507(2)$ & $\mathrm{C} 7 \mathrm{~B}-\mathrm{C} 8 \mathrm{~B}$ & $1.508(2)$ \\
\hline $\mathrm{C} 7 \mathrm{~A}-\mathrm{C} 8 \mathrm{~A}$ & $1.511(2)$ & $\mathrm{C} 7 \mathrm{~B}-\mathrm{C} 9 \mathrm{~B}$ & $1.516(3)$ \\
\hline $\mathrm{C} 7 \mathrm{~A}-\mathrm{H} 7 \mathrm{~A}$ & 1.0000 & $\mathrm{C} 7 \mathrm{~B}-\mathrm{H} 7 \mathrm{~B}$ & 1.0000 \\
\hline $\mathrm{C} 8 \mathrm{~A}-\mathrm{H} 8 \mathrm{AA}$ & 0.9800 & $\mathrm{C} 8 \mathrm{~B}-\mathrm{H} 8 \mathrm{BA}$ & 0.9800 \\
\hline $\mathrm{C} 8 \mathrm{~A}-\mathrm{H} 8 \mathrm{AB}$ & 0.9800 & $\mathrm{C} 8 \mathrm{~B}-\mathrm{H} 8 \mathrm{BB}$ & 0.9800 \\
\hline $\mathrm{C} 8 \mathrm{~A}-\mathrm{H} 8 \mathrm{AC}$ & 0.9800 & $\mathrm{C} 8 \mathrm{~B}-\mathrm{H} 8 \mathrm{BC}$ & 0.9800 \\
\hline C9A-H9AA & 0.9800 & C9B-H9BA & 0.9800 \\
\hline $\mathrm{C} 9 \mathrm{~A}-\mathrm{H} 9 \mathrm{AB}$ & 0.9800 & C9B-H9BB & 0.9800 \\
\hline $\mathrm{C} 9 \mathrm{~A}-\mathrm{H} 9 \mathrm{AC}$ & 0.9800 & C9B-H9BC & 0.9800 \\
\hline $\mathrm{C} 10 \mathrm{~A}-\mathrm{C} 11 \mathrm{~A}$ & $1.517(2)$ & $\mathrm{C} 10 \mathrm{~B}-\mathrm{C} 11 \mathrm{~B}$ & $1.513(2)$ \\
\hline $\mathrm{C} 10 \mathrm{~A}-\mathrm{C} 12 \mathrm{~A}$ & $1.518(2)$ & $\mathrm{C} 10 \mathrm{~B}-\mathrm{C} 12 \mathrm{~B}$ & $1.522(2)$ \\
\hline $\mathrm{C} 10 \mathrm{~A}-\mathrm{H} 10 \mathrm{~A}$ & 1.0000 & $\mathrm{C} 10 \mathrm{~B}-\mathrm{H} 10 \mathrm{~B}$ & 1.0000 \\
\hline $\mathrm{C} 11 \mathrm{~A}-\mathrm{H} 11 \mathrm{~A}$ & 0.9800 & $\mathrm{C} 11 \mathrm{~B}-\mathrm{H} 11 \mathrm{D}$ & 0.9800 \\
\hline $\mathrm{C} 11 \mathrm{~A}-\mathrm{H} 11 \mathrm{~B}$ & 0.9800 & $\mathrm{C} 11 \mathrm{~B}-\mathrm{H} 11 \mathrm{E}$ & 0.9800 \\
\hline $\mathrm{C} 11 \mathrm{~A}-\mathrm{H} 11 \mathrm{C}$ & 0.9800 & $\mathrm{C} 11 \mathrm{~B}-\mathrm{H} 11 \mathrm{~F}$ & 0.9800 \\
\hline $\mathrm{C} 12 \mathrm{~A}-\mathrm{H} 12 \mathrm{~A}$ & 0.9800 & $\mathrm{C} 12 \mathrm{~B}-\mathrm{H} 12 \mathrm{D}$ & 0.9800 \\
\hline $\mathrm{C} 12 \mathrm{~A}-\mathrm{H} 12 \mathrm{~B}$ & 0.9800 & $\mathrm{C} 12 \mathrm{~B}-\mathrm{H} 12 \mathrm{E}$ & 0.9800 \\
\hline $\mathrm{C} 12 \mathrm{~A}-\mathrm{H} 12 \mathrm{C}$ & 0.9800 & $\mathrm{C} 12 \mathrm{~B}-\mathrm{H} 12 \mathrm{~F}$ & 0.9800 \\
\hline $\mathrm{C} 13 \mathrm{~A}-\mathrm{C} 14 \mathrm{~A}$ & $1.399(2)$ & $\mathrm{C} 13 \mathrm{~B}-\mathrm{C} 14 \mathrm{~B}$ & $1.396(2)$ \\
\hline $\mathrm{C} 13 \mathrm{~A}-\mathrm{C} 18 \mathrm{~A}$ & $1.403(2)$ & $\mathrm{C} 13 \mathrm{~B}-\mathrm{C} 18 \mathrm{~B}$ & $1.402(2)$ \\
\hline $\mathrm{C} 14 \mathrm{~A}-\mathrm{C} 15 \mathrm{~A}$ & $1.379(2)$ & $\mathrm{C} 14 \mathrm{~B}-\mathrm{C} 15 \mathrm{~B}$ & $1.385(2)$ \\
\hline $\mathrm{C} 14 \mathrm{~A}-\mathrm{H} 14 \mathrm{~A}$ & 0.9500 & $\mathrm{C} 14 \mathrm{~B}-\mathrm{H} 14 \mathrm{~B}$ & 0.9500 \\
\hline $\mathrm{C} 15 \mathrm{~A}-\mathrm{C} 16 \mathrm{~A}$ & $1.387(2)$ & $\mathrm{C} 15 \mathrm{~B}-\mathrm{C} 16 \mathrm{~B}$ & $1.390(2)$ \\
\hline $\mathrm{C} 15 \mathrm{~A}-\mathrm{H} 15 \mathrm{~A}$ & 0.9500 & $\mathrm{C} 15 \mathrm{~B}-\mathrm{H} 15 \mathrm{~B}$ & 0.9500 \\
\hline $\mathrm{C} 16 \mathrm{~A}-\mathrm{C} 17 \mathrm{~A}$ & $1.389(2)$ & $\mathrm{C} 16 \mathrm{~B}-\mathrm{C} 17 \mathrm{~B}$ & $1.387(2)$ \\
\hline $\mathrm{C} 16 \mathrm{~A}-\mathrm{H} 16 \mathrm{~A}$ & 0.9500 & $\mathrm{C} 16 \mathrm{~B}-\mathrm{H} 16 \mathrm{~B}$ & 0.9500 \\
\hline $\mathrm{C} 17 \mathrm{~A}-\mathrm{C} 18 \mathrm{~A}$ & $1.397(2)$ & $\mathrm{C} 17 \mathrm{~B}-\mathrm{C} 18 \mathrm{~B}$ & $1.402(2)$ \\
\hline $\mathrm{C} 17 \mathrm{~A}-\mathrm{H} 17 \mathrm{~A}$ & 0.9500 & $\mathrm{C} 17 \mathrm{~B}-\mathrm{H} 17 \mathrm{~B}$ & 0.9500 \\
\hline $\mathrm{C} 19 \mathrm{~A}-\mathrm{C} 20 \mathrm{~A}$ & $1.527(2)$ & $\mathrm{C} 19 \mathrm{~B}-\mathrm{C} 20 \mathrm{~B}$ & $1.529(2)$ \\
\hline $\mathrm{C} 19 \mathrm{~A}-\mathrm{C} 24 \mathrm{~A}$ & $1.532(2)$ & $\mathrm{C} 19 \mathrm{~B}-\mathrm{C} 24 \mathrm{~B}$ & $1.538(2)$ \\
\hline C19A-H19A & 1.0000 & $\mathrm{C} 19 \mathrm{~B}-\mathrm{H} 19 \mathrm{~B}$ & 1.0000 \\
\hline $\mathrm{C} 20 \mathrm{~A}-\mathrm{C} 21 \mathrm{~A}$ & $1.534(3)$ & $\mathrm{C} 20 \mathrm{~B}-\mathrm{C} 21 \mathrm{~B}$ & $1.528(2)$ \\
\hline $\mathrm{C} 20 \mathrm{~A}-\mathrm{H} 20 \mathrm{~A}$ & 0.9900 & $\mathrm{C} 20 \mathrm{~B}-\mathrm{H} 20 \mathrm{C}$ & 0.9900 \\
\hline $\mathrm{C} 20 \mathrm{~A}-\mathrm{H} 20 \mathrm{~B}$ & 0.9900 & $\mathrm{C} 20 \mathrm{~B}-\mathrm{H} 20 \mathrm{D}$ & 0.9900 \\
\hline $\mathrm{C} 21 \mathrm{~A}-\mathrm{C} 22 \mathrm{~A}$ & $1.520(3)$ & $\mathrm{C} 21 \mathrm{~B}-\mathrm{C} 22 \mathrm{~B}$ & $1.521(3)$ \\
\hline $\mathrm{C} 21 \mathrm{~A}-\mathrm{H} 21 \mathrm{~A}$ & 0.9900 & $\mathrm{C} 21 \mathrm{~B}-\mathrm{H} 21 \mathrm{C}$ & 0.9900 \\
\hline
\end{tabular}




\begin{tabular}{|c|c|c|c|}
\hline $\mathrm{C} 21 \mathrm{~A}-\mathrm{H} 21 \mathrm{~B}$ & 0.9900 & $\mathrm{C} 21 \mathrm{~B}-\mathrm{H} 21 \mathrm{D}$ & 0.9900 \\
\hline $\mathrm{C} 22 \mathrm{~A}-\mathrm{C} 23 \mathrm{~A}$ & $1.518(3)$ & $\mathrm{C} 22 \mathrm{~B}-\mathrm{C} 23 \mathrm{~B}$ & $1.518(3)$ \\
\hline $\mathrm{C} 22 \mathrm{~A}-\mathrm{H} 22 \mathrm{~A}$ & 0.9900 & $\mathrm{C} 22 \mathrm{~B}-\mathrm{H} 22 \mathrm{C}$ & 0.9900 \\
\hline $\mathrm{C} 22 \mathrm{~A}-\mathrm{H} 22 \mathrm{~B}$ & 0.9900 & $\mathrm{C} 22 \mathrm{~B}-\mathrm{H} 22 \mathrm{D}$ & 0.9900 \\
\hline $\mathrm{C} 23 \mathrm{~A}-\mathrm{C} 24 \mathrm{~A}$ & $1.532(3)$ & $\mathrm{C} 23 \mathrm{~B}-\mathrm{C} 24 \mathrm{~B}$ & $1.528(2)$ \\
\hline $\mathrm{C} 23 \mathrm{~A}-\mathrm{H} 23 \mathrm{~A}$ & 0.9900 & $\mathrm{C} 23 \mathrm{~B}-\mathrm{H} 23 \mathrm{C}$ & 0.9900 \\
\hline $\mathrm{C} 23 \mathrm{~A}-\mathrm{H} 23 \mathrm{~B}$ & 0.9900 & $\mathrm{C} 23 \mathrm{~B}-\mathrm{H} 23 \mathrm{D}$ & 0.9900 \\
\hline $\mathrm{C} 24 \mathrm{~A}-\mathrm{H} 24 \mathrm{~A}$ & 0.9900 & $\mathrm{C} 24 \mathrm{~B}-\mathrm{H} 24 \mathrm{C}$ & 0.9900 \\
\hline $\mathrm{C} 24 \mathrm{~A}-\mathrm{H} 24 \mathrm{~B}$ & 0.9900 & $\mathrm{C} 24 \mathrm{~B}-\mathrm{H} 24 \mathrm{D}$ & 0.9900 \\
\hline $\mathrm{C} 25 \mathrm{~A}-\mathrm{C} 26 \mathrm{~A}$ & $1.535(2)$ & $\mathrm{C} 25 \mathrm{~B}-\mathrm{C} 30 \mathrm{~B}$ & $1.535(2)$ \\
\hline $\mathrm{C} 25 \mathrm{~A}-\mathrm{C} 30 \mathrm{~A}$ & $1.541(2)$ & $\mathrm{C} 25 \mathrm{~B}-\mathrm{C} 26 \mathrm{~B}$ & $1.544(2)$ \\
\hline $\mathrm{C} 25 \mathrm{~A}-\mathrm{H} 25 \mathrm{~A}$ & 1.0000 & $\mathrm{C} 25 \mathrm{~B}-\mathrm{H} 25 \mathrm{~B}$ & 1.0000 \\
\hline $\mathrm{C} 26 \mathrm{~A}-\mathrm{C} 27 \mathrm{~A}$ & $1.531(2)$ & $\mathrm{C} 26 \mathrm{~B}-\mathrm{C} 27 \mathrm{~B}$ & $1.528(2)$ \\
\hline $\mathrm{C} 26 \mathrm{~A}-\mathrm{H} 26 \mathrm{~A}$ & 0.9900 & $\mathrm{C} 26 \mathrm{~B}-\mathrm{H} 26 \mathrm{C}$ & 0.9900 \\
\hline $\mathrm{C} 26 \mathrm{~A}-\mathrm{H} 26 \mathrm{~B}$ & 0.9900 & $\mathrm{C} 26 \mathrm{~B}-\mathrm{H} 26 \mathrm{D}$ & 0.9900 \\
\hline $\mathrm{C} 27 \mathrm{~A}-\mathrm{C} 28 \mathrm{~A}$ & $1.531(2)$ & $\mathrm{C} 27 \mathrm{~B}-\mathrm{C} 28 \mathrm{~B}$ & $1.529(3)$ \\
\hline $\mathrm{C} 27 \mathrm{~A}-\mathrm{H} 27 \mathrm{~A}$ & 0.9900 & $\mathrm{C} 27 \mathrm{~B}-\mathrm{H} 27 \mathrm{C}$ & 0.9900 \\
\hline $\mathrm{C} 27 \mathrm{~A}-\mathrm{H} 27 \mathrm{~B}$ & 0.9900 & $\mathrm{C} 27 \mathrm{~B}-\mathrm{H} 27 \mathrm{D}$ & 0.9900 \\
\hline $\mathrm{C} 28 \mathrm{~A}-\mathrm{C} 29 \mathrm{~A}$ & $1.522(2)$ & $\mathrm{C} 28 \mathrm{~B}-\mathrm{C} 29 \mathrm{~B}$ & $1.527(2)$ \\
\hline $\mathrm{C} 28 \mathrm{~A}-\mathrm{H} 28 \mathrm{~A}$ & 0.9900 & $\mathrm{C} 28 \mathrm{~B}-\mathrm{H} 28 \mathrm{C}$ & 0.9900 \\
\hline $\mathrm{C} 28 \mathrm{~A}-\mathrm{H} 28 \mathrm{~B}$ & 0.9900 & $\mathrm{C} 28 \mathrm{~B}-\mathrm{H} 28 \mathrm{D}$ & 0.9900 \\
\hline $\mathrm{C} 29 \mathrm{~A}-\mathrm{C} 30 \mathrm{~A}$ & $1.528(2)$ & $\mathrm{C} 29 \mathrm{~B}-\mathrm{C} 30 \mathrm{~B}$ & $1.532(2)$ \\
\hline $\mathrm{C} 29 \mathrm{~A}-\mathrm{H} 29 \mathrm{~A}$ & 0.9900 & $\mathrm{C} 29 \mathrm{~B}-\mathrm{H} 29 \mathrm{C}$ & 0.9900 \\
\hline $\mathrm{C} 29 \mathrm{~A}-\mathrm{H} 29 \mathrm{~B}$ & 0.9900 & $\mathrm{C} 29 \mathrm{~B}-\mathrm{H} 29 \mathrm{D}$ & 0.9900 \\
\hline $\mathrm{C} 30 \mathrm{~A}-\mathrm{H} 30 \mathrm{~A}$ & 0.9900 & $\mathrm{C} 30 \mathrm{~B}-\mathrm{H} 30 \mathrm{C}$ & 0.9900 \\
\hline $\mathrm{C} 30 \mathrm{~A}-\mathrm{H} 30 \mathrm{~B}$ & 0.9900 & $\mathrm{C} 30 \mathrm{~B}-\mathrm{H} 30 \mathrm{D}$ & 0.9900 \\
\hline $\mathrm{C} 18 \mathrm{~A}-\mathrm{P} 1 \mathrm{~A}-\mathrm{C} 25 \mathrm{~A}$ & $101.31(7)$ & $\mathrm{C} 18 \mathrm{~B}-\mathrm{P} 1 \mathrm{~B}-\mathrm{C} 25 \mathrm{~B}$ & $101.86(8)$ \\
\hline $\mathrm{C} 18 \mathrm{~A}-\mathrm{P} 1 \mathrm{~A}-\mathrm{C} 19 \mathrm{~A}$ & $98.31(7)$ & $\mathrm{C} 18 \mathrm{~B}-\mathrm{P} 1 \mathrm{~B}-\mathrm{C} 19 \mathrm{~B}$ & $97.03(7)$ \\
\hline $\mathrm{C} 25 \mathrm{~A}-\mathrm{P} 1 \mathrm{~A}-\mathrm{C} 19 \mathrm{~A}$ & $106.07(8)$ & $\mathrm{C} 25 \mathrm{~B}-\mathrm{P} 1 \mathrm{~B}-\mathrm{C} 19 \mathrm{~B}$ & $105.46(8)$ \\
\hline $\mathrm{C} 3 \mathrm{~A}-\mathrm{O} 1 \mathrm{~A}-\mathrm{C} 7 \mathrm{~A}$ & $119.19(12)$ & $\mathrm{C} 3 \mathrm{~B}-\mathrm{O} 1 \mathrm{~B}-\mathrm{C} 7 \mathrm{~B}$ & $119.71(13)$ \\
\hline $\mathrm{C} 5 \mathrm{~A}-\mathrm{O} 2 \mathrm{~A}-\mathrm{C} 10 \mathrm{~A}$ & $120.37(13)$ & $\mathrm{C} 5 \mathrm{~B}-\mathrm{O} 2 \mathrm{~B}-\mathrm{C} 10 \mathrm{~B}$ & $119.60(13)$ \\
\hline $\mathrm{C} 2 \mathrm{~A}-\mathrm{C} 1 \mathrm{~A}-\mathrm{C} 6 \mathrm{~A}$ & $121.85(16)$ & $\mathrm{C} 2 \mathrm{~B}-\mathrm{C} 1 \mathrm{~B}-\mathrm{C} 6 \mathrm{~B}$ & $121.67(16)$ \\
\hline $\mathrm{C} 2 \mathrm{~A}-\mathrm{C} 1 \mathrm{~A}-\mathrm{H} 1 \mathrm{~A}$ & 119.1 & $\mathrm{C} 2 \mathrm{~B}-\mathrm{C} 1 \mathrm{~B}-\mathrm{H} 1 \mathrm{~B}$ & 119.2 \\
\hline $\mathrm{C} 6 \mathrm{~A}-\mathrm{C} 1 \mathrm{~A}-\mathrm{H} 1 \mathrm{~A}$ & 119.1 & $\mathrm{C} 6 \mathrm{~B}-\mathrm{C} 1 \mathrm{~B}-\mathrm{H} 1 \mathrm{~B}$ & 119.2 \\
\hline $\mathrm{C} 1 \mathrm{~A}-\mathrm{C} 2 \mathrm{~A}-\mathrm{C} 3 \mathrm{~A}$ & $118.90(16)$ & $\mathrm{C} 1 \mathrm{~B}-\mathrm{C} 2 \mathrm{~B}-\mathrm{C} 3 \mathrm{~B}$ & $118.66(16)$ \\
\hline $\mathrm{C} 1 \mathrm{~A}-\mathrm{C} 2 \mathrm{~A}-\mathrm{H} 2 \mathrm{~A}$ & 120.6 & $\mathrm{C} 1 \mathrm{~B}-\mathrm{C} 2 \mathrm{~B}-\mathrm{H} 2 \mathrm{~B}$ & 120.7 \\
\hline $\mathrm{C} 3 \mathrm{~A}-\mathrm{C} 2 \mathrm{~A}-\mathrm{H} 2 \mathrm{~A}$ & 120.6 & $\mathrm{C} 3 \mathrm{~B}-\mathrm{C} 2 \mathrm{~B}-\mathrm{H} 2 \mathrm{~B}$ & 120.7 \\
\hline $\mathrm{O} 1 \mathrm{~A}-\mathrm{C} 3 \mathrm{~A}-\mathrm{C} 2 \mathrm{~A}$ & $124.80(15)$ & $\mathrm{O} 1 \mathrm{~B}-\mathrm{C} 3 \mathrm{~B}-\mathrm{C} 2 \mathrm{~B}$ & $124.58(15)$ \\
\hline $\mathrm{O} 1 \mathrm{~A}-\mathrm{C} 3 \mathrm{~A}-\mathrm{C} 4 \mathrm{~A}$ & $114.40(14)$ & $\mathrm{O} 1 \mathrm{~B}-\mathrm{C} 3 \mathrm{~B}-\mathrm{C} 4 \mathrm{~B}$ & $114.10(14)$ \\
\hline $\mathrm{C} 2 \mathrm{~A}-\mathrm{C} 3 \mathrm{~A}-\mathrm{C} 4 \mathrm{~A}$ & $120.76(15)$ & $\mathrm{C} 2 \mathrm{~B}-\mathrm{C} 3 \mathrm{~B}-\mathrm{C} 4 \mathrm{~B}$ & $121.29(15)$ \\
\hline $\mathrm{C} 5 \mathrm{~A}-\mathrm{C} 4 \mathrm{~A}-\mathrm{C} 3 \mathrm{~A}$ & $118.97(15)$ & $\mathrm{C} 3 \mathrm{~B}-\mathrm{C} 4 \mathrm{~B}-\mathrm{C} 5 \mathrm{~B}$ & $118.52(15)$ \\
\hline $\mathrm{C} 5 \mathrm{~A}-\mathrm{C} 4 \mathrm{~A}-\mathrm{C} 13 \mathrm{~A}$ & $120.65(15)$ & $\mathrm{C} 3 \mathrm{~B}-\mathrm{C} 4 \mathrm{~B}-\mathrm{C} 13 \mathrm{~B}$ & $120.34(14)$ \\
\hline $\mathrm{C} 3 \mathrm{~A}-\mathrm{C} 4 \mathrm{~A}-\mathrm{C} 13 \mathrm{~A}$ & $120.36(15)$ & $\mathrm{C} 5 \mathrm{~B}-\mathrm{C} 4 \mathrm{~B}-\mathrm{C} 13 \mathrm{~B}$ & $121.08(14)$ \\
\hline $\mathrm{O} 2 \mathrm{~A}-\mathrm{C} 5 \mathrm{~A}-\mathrm{C} 6 \mathrm{~A}$ & $125.33(15)$ & $\mathrm{O} 2 \mathrm{~B}-\mathrm{C} 5 \mathrm{~B}-\mathrm{C} 6 \mathrm{~B}$ & $124.82(15)$ \\
\hline $\mathrm{O} 2 \mathrm{~A}-\mathrm{C} 5 \mathrm{~A}-\mathrm{C} 4 \mathrm{~A}$ & $113.90(14)$ & $\mathrm{O} 2 \mathrm{~B}-\mathrm{C} 5 \mathrm{~B}-\mathrm{C} 4 \mathrm{~B}$ & $114.52(14)$ \\
\hline $\mathrm{C} 6 \mathrm{~A}-\mathrm{C} 5 \mathrm{~A}-\mathrm{C} 4 \mathrm{~A}$ & $120.76(16)$ & $\mathrm{C} 6 \mathrm{~B}-\mathrm{C} 5 \mathrm{~B}-\mathrm{C} 4 \mathrm{~B}$ & $120.66(15)$ \\
\hline $\mathrm{C} 1 \mathrm{~A}-\mathrm{C} 6 \mathrm{~A}-\mathrm{C} 5 \mathrm{~A}$ & $118.68(16)$ & $\mathrm{C} 1 \mathrm{~B}-\mathrm{C} 6 \mathrm{~B}-\mathrm{C} 5 \mathrm{~B}$ & $119.18(16)$ \\
\hline
\end{tabular}




\begin{tabular}{|c|c|}
\hline $\mathrm{C} 1 \mathrm{~A}-\mathrm{C} 6 \mathrm{~A}-\mathrm{H} 6 \mathrm{~A}$ & 120.7 \\
\hline $\mathrm{C} 5 \mathrm{~A}-\mathrm{C} 6 \mathrm{~A}-\mathrm{H} 6 \mathrm{~A}$ & 120.7 \\
\hline $\mathrm{O} 1 \mathrm{~A}-\mathrm{C} 7 \mathrm{~A}-\mathrm{C} 9 \mathrm{~A}$ & $110.12(14)$ \\
\hline $\mathrm{O} 1 \mathrm{~A}-\mathrm{C} 7 \mathrm{~A}-\mathrm{C} 8 \mathrm{~A}$ & $104.46(13)$ \\
\hline $\mathrm{C} 9 \mathrm{~A}-\mathrm{C} 7 \mathrm{~A}-\mathrm{C} 8 \mathrm{~A}$ & $113.08(15)$ \\
\hline $\mathrm{O} 1 \mathrm{~A}-\mathrm{C} 7 \mathrm{~A}-\mathrm{H} 7 \mathrm{~A}$ & 109.7 \\
\hline $\mathrm{C} 9 \mathrm{~A}-\mathrm{C} 7 \mathrm{~A}-\mathrm{H} 7 \mathrm{~A}$ & 109.7 \\
\hline $\mathrm{C} 8 \mathrm{~A}-\mathrm{C} 7 \mathrm{~A}-\mathrm{H} 7 \mathrm{~A}$ & 109.7 \\
\hline $\mathrm{C} 7 \mathrm{~A}-\mathrm{C} 8 \mathrm{~A}-\mathrm{H} 8 \mathrm{AA}$ & 109.5 \\
\hline $\mathrm{C} 7 \mathrm{~A}-\mathrm{C} 8 \mathrm{~A}-\mathrm{H} 8 \mathrm{AB}$ & 109.5 \\
\hline $\mathrm{H} 8 \mathrm{AA}-\mathrm{C} 8 \mathrm{~A}-\mathrm{H} 8 \mathrm{AB}$ & 109.5 \\
\hline $\mathrm{C} 7 \mathrm{~A}-\mathrm{C} 8 \mathrm{~A}-\mathrm{H} 8 \mathrm{AC}$ & 109.5 \\
\hline $\mathrm{H} 8 \mathrm{AA}-\mathrm{C} 8 \mathrm{~A}-\mathrm{H} 8 \mathrm{AC}$ & 109.5 \\
\hline $\mathrm{H} 8 \mathrm{AB}-\mathrm{C} 8 \mathrm{~A}-\mathrm{H} 8 \mathrm{AC}$ & 109.5 \\
\hline $\mathrm{C} 7 \mathrm{~A}-\mathrm{C} 9 \mathrm{~A}-\mathrm{H} 9 \mathrm{AA}$ & 109.5 \\
\hline $\mathrm{C} 7 \mathrm{~A}-\mathrm{C} 9 \mathrm{~A}-\mathrm{H} 9 \mathrm{AB}$ & 109.5 \\
\hline H9AA-C9A-H9AB & 109.5 \\
\hline $\mathrm{C} 7 \mathrm{~A}-\mathrm{C} 9 \mathrm{~A}-\mathrm{H} 9 \mathrm{AC}$ & 109.5 \\
\hline $\mathrm{H} 9 \mathrm{AA}-\mathrm{C} 9 \mathrm{~A}-\mathrm{H} 9 \mathrm{AC}$ & 109.5 \\
\hline $\mathrm{H} 9 \mathrm{AB}-\mathrm{C} 9 \mathrm{~A}-\mathrm{H} 9 \mathrm{AC}$ & 109.5 \\
\hline $\mathrm{O} 2 \mathrm{~A}-\mathrm{C} 10 \mathrm{~A}-\mathrm{C} 11 \mathrm{~A}$ & $104.29(13)$ \\
\hline $\mathrm{O} 2 \mathrm{~A}-\mathrm{C} 10 \mathrm{~A}-\mathrm{C} 12 \mathrm{~A}$ & $111.20(14)$ \\
\hline $\mathrm{C} 11 \mathrm{~A}-\mathrm{C} 10 \mathrm{~A}-\mathrm{C} 12 \mathrm{~A}$ & $111.12(15)$ \\
\hline $\mathrm{O} 2 \mathrm{~A}-\mathrm{C} 10 \mathrm{~A}-\mathrm{H} 10 \mathrm{~A}$ & 110.0 \\
\hline $\mathrm{C} 11 \mathrm{~A}-\mathrm{C} 10 \mathrm{~A}-\mathrm{H} 10 \mathrm{~A}$ & 110.0 \\
\hline $\mathrm{C} 12 \mathrm{~A}-\mathrm{C} 10 \mathrm{~A}-\mathrm{H} 10 \mathrm{~A}$ & 110.0 \\
\hline $\mathrm{C} 10 \mathrm{~A}-\mathrm{C} 11 \mathrm{~A}-\mathrm{H} 11 \mathrm{~A}$ & 109.5 \\
\hline $\mathrm{C} 10 \mathrm{~A}-\mathrm{C} 11 \mathrm{~A}-\mathrm{H} 11 \mathrm{~B}$ & 109.5 \\
\hline $\mathrm{H} 11 \mathrm{~A}-\mathrm{C} 11 \mathrm{~A}-\mathrm{H} 11 \mathrm{~B}$ & 109.5 \\
\hline $\mathrm{C} 10 \mathrm{~A}-\mathrm{C} 11 \mathrm{~A}-\mathrm{H} 11 \mathrm{C}$ & 109.5 \\
\hline $\mathrm{H} 11 \mathrm{~A}-\mathrm{C} 11 \mathrm{~A}-\mathrm{H} 11 \mathrm{C}$ & 109.5 \\
\hline $\mathrm{H} 11 \mathrm{~B}-\mathrm{C} 11 \mathrm{~A}-\mathrm{H} 11 \mathrm{C}$ & 109.5 \\
\hline $\mathrm{C} 10 \mathrm{~A}-\mathrm{C} 12 \mathrm{~A}-\mathrm{H} 12 \mathrm{~A}$ & 109.5 \\
\hline $\mathrm{C} 10 \mathrm{~A}-\mathrm{C} 12 \mathrm{~A}-\mathrm{H} 12 \mathrm{~B}$ & 109.5 \\
\hline $\mathrm{H} 12 \mathrm{~A}-\mathrm{C} 12 \mathrm{~A}-\mathrm{H} 12 \mathrm{~B}$ & 109.5 \\
\hline $\mathrm{C} 10 \mathrm{~A}-\mathrm{C} 12 \mathrm{~A}-\mathrm{H} 12 \mathrm{C}$ & 109.5 \\
\hline $\mathrm{H} 12 \mathrm{~A}-\mathrm{C} 12 \mathrm{~A}-\mathrm{H} 12 \mathrm{C}$ & 109.5 \\
\hline $\mathrm{H} 12 \mathrm{~B}-\mathrm{C} 12 \mathrm{~A}-\mathrm{H} 12 \mathrm{C}$ & 109.5 \\
\hline $\mathrm{C} 14 \mathrm{~A}-\mathrm{C} 13 \mathrm{~A}-\mathrm{C} 18 \mathrm{~A}$ & $119.78(15)$ \\
\hline $\mathrm{C} 14 \mathrm{~A}-\mathrm{C} 13 \mathrm{~A}-\mathrm{C} 4 \mathrm{~A}$ & $118.46(15)$ \\
\hline $\mathrm{C} 18 \mathrm{~A}-\mathrm{C} 13 \mathrm{~A}-\mathrm{C} 4 \mathrm{~A}$ & $121.76(14)$ \\
\hline $\mathrm{C} 15 \mathrm{~A}-\mathrm{C} 14 \mathrm{~A}-\mathrm{C} 13 \mathrm{~A}$ & $121.16(16)$ \\
\hline $\mathrm{C} 15 \mathrm{~A}-\mathrm{C} 14 \mathrm{~A}-\mathrm{H} 14 \mathrm{~A}$ & 119.4 \\
\hline $\mathrm{C} 13 \mathrm{~A}-\mathrm{C} 14 \mathrm{~A}-\mathrm{H} 14 \mathrm{~A}$ & 119.4 \\
\hline $\mathrm{C} 14 \mathrm{~A}-\mathrm{C} 15 \mathrm{~A}-\mathrm{C} 16 \mathrm{~A}$ & $119.48(16)$ \\
\hline $\mathrm{C} 14 \mathrm{~A}-\mathrm{C} 15 \mathrm{~A}-\mathrm{H} 15 \mathrm{~A}$ & 120.3 \\
\hline $\mathrm{C} 16 \mathrm{~A}-\mathrm{C} 15 \mathrm{~A}-\mathrm{H} 15 \mathrm{~A}$ & 120.3 \\
\hline $\mathrm{C} 15 \mathrm{~A}-\mathrm{C} 16 \mathrm{~A}-\mathrm{C} 17 \mathrm{~A}$ & $119.91(15)$ \\
\hline
\end{tabular}

\begin{tabular}{|c|c|}
\hline $\mathrm{C} 1 \mathrm{~B}-\mathrm{C} 6 \mathrm{~B}-\mathrm{H} 6 \mathrm{~B}$ & 120.4 \\
\hline $\mathrm{C} 5 \mathrm{~B}-\mathrm{C} 6 \mathrm{~B}-\mathrm{H} 6 \mathrm{~B}$ & 120.4 \\
\hline $\mathrm{O} 1 \mathrm{~B}-\mathrm{C} 7 \mathrm{~B}-\mathrm{C} 8 \mathrm{~B}$ & $104.22(14)$ \\
\hline $\mathrm{O} 1 \mathrm{~B}-\mathrm{C} 7 \mathrm{~B}-\mathrm{C} 9 \mathrm{~B}$ & $110.32(14)$ \\
\hline $\mathrm{C} 8 \mathrm{~B}-\mathrm{C} 7 \mathrm{~B}-\mathrm{C} 9 \mathrm{~B}$ & $113.21(16)$ \\
\hline $\mathrm{O} 1 \mathrm{~B}-\mathrm{C} 7 \mathrm{~B}-\mathrm{H} 7 \mathrm{~B}$ & 109.6 \\
\hline $\mathrm{C} 8 \mathrm{~B}-\mathrm{C} 7 \mathrm{~B}-\mathrm{H} 7 \mathrm{~B}$ & 109.6 \\
\hline $\mathrm{C} 9 \mathrm{~B}-\mathrm{C} 7 \mathrm{~B}-\mathrm{H} 7 \mathrm{~B}$ & 109.6 \\
\hline $\mathrm{C} 7 \mathrm{~B}-\mathrm{C} 8 \mathrm{~B}-\mathrm{H} 8 \mathrm{BA}$ & 109.5 \\
\hline $\mathrm{C} 7 \mathrm{~B}-\mathrm{C} 8 \mathrm{~B}-\mathrm{H} 8 \mathrm{BB}$ & 109.5 \\
\hline $\mathrm{H} 8 \mathrm{BA}-\mathrm{C} 8 \mathrm{~B}-\mathrm{H} 8 \mathrm{BB}$ & 109.5 \\
\hline $\mathrm{C} 7 \mathrm{~B}-\mathrm{C} 8 \mathrm{~B}-\mathrm{H} 8 \mathrm{BC}$ & 109.5 \\
\hline $\mathrm{H} 8 \mathrm{BA}-\mathrm{C} 8 \mathrm{~B}-\mathrm{H} 8 \mathrm{BC}$ & 109.5 \\
\hline $\mathrm{H} 8 \mathrm{BB}-\mathrm{C} 8 \mathrm{~B}-\mathrm{H} 8 \mathrm{BC}$ & 109.5 \\
\hline $\mathrm{C} 7 \mathrm{~B}-\mathrm{C} 9 \mathrm{~B}-\mathrm{H} 9 \mathrm{BA}$ & 109.5 \\
\hline $\mathrm{C} 7 \mathrm{~B}-\mathrm{C} 9 \mathrm{~B}-\mathrm{H} 9 \mathrm{BB}$ & 109.5 \\
\hline H9BA-C9B-H9BB & 109.5 \\
\hline $\mathrm{C} 7 \mathrm{~B}-\mathrm{C} 9 \mathrm{~B}-\mathrm{H} 9 \mathrm{BC}$ & 109.5 \\
\hline $\mathrm{H} 9 \mathrm{BA}-\mathrm{C} 9 \mathrm{~B}-\mathrm{H} 9 \mathrm{BC}$ & 109.5 \\
\hline $\mathrm{H} 9 \mathrm{BB}-\mathrm{C} 9 \mathrm{~B}-\mathrm{H} 9 \mathrm{BC}$ & 109.5 \\
\hline $\mathrm{O} 2 \mathrm{~B}-\mathrm{C} 10 \mathrm{~B}-\mathrm{C} 11 \mathrm{~B}$ & $105.04(14)$ \\
\hline $\mathrm{O} 2 \mathrm{~B}-\mathrm{C} 10 \mathrm{~B}-\mathrm{C} 12 \mathrm{~B}$ & $111.04(14)$ \\
\hline $\mathrm{C} 11 \mathrm{~B}-\mathrm{C} 10 \mathrm{~B}-\mathrm{C} 12 \mathrm{~B}$ & $111.61(15)$ \\
\hline $\mathrm{O} 2 \mathrm{~B}-\mathrm{C} 10 \mathrm{~B}-\mathrm{H} 10 \mathrm{~B}$ & 109.7 \\
\hline $\mathrm{C} 11 \mathrm{~B}-\mathrm{C} 10 \mathrm{~B}-\mathrm{H} 10 \mathrm{~B}$ & 109.7 \\
\hline $\mathrm{C} 12 \mathrm{~B}-\mathrm{C} 10 \mathrm{~B}-\mathrm{H} 10 \mathrm{~B}$ & 109.7 \\
\hline $\mathrm{C} 10 \mathrm{~B}-\mathrm{C} 11 \mathrm{~B}-\mathrm{H} 11 \mathrm{D}$ & 109.5 \\
\hline $\mathrm{C} 10 \mathrm{~B}-\mathrm{C} 11 \mathrm{~B}-\mathrm{H} 11 \mathrm{E}$ & 109.5 \\
\hline $\mathrm{H} 11 \mathrm{D}-\mathrm{C} 11 \mathrm{~B}-\mathrm{H} 11 \mathrm{E}$ & 109.5 \\
\hline $\mathrm{C} 10 \mathrm{~B}-\mathrm{C} 11 \mathrm{~B}-\mathrm{H} 11 \mathrm{~F}$ & 109.5 \\
\hline $\mathrm{H} 11 \mathrm{D}-\mathrm{C} 11 \mathrm{~B}-\mathrm{H} 11 \mathrm{~F}$ & 109.5 \\
\hline $\mathrm{H} 11 \mathrm{E}-\mathrm{C} 11 \mathrm{~B}-\mathrm{H} 11 \mathrm{~F}$ & 109.5 \\
\hline $\mathrm{C} 10 \mathrm{~B}-\mathrm{C} 12 \mathrm{~B}-\mathrm{H} 12 \mathrm{D}$ & 109.5 \\
\hline $\mathrm{C} 10 \mathrm{~B}-\mathrm{C} 12 \mathrm{~B}-\mathrm{H} 12 \mathrm{E}$ & 109.5 \\
\hline $\mathrm{H} 12 \mathrm{D}-\mathrm{C} 12 \mathrm{~B}-\mathrm{H} 12 \mathrm{E}$ & 109.5 \\
\hline $\mathrm{C} 10 \mathrm{~B}-\mathrm{C} 12 \mathrm{~B}-\mathrm{H} 12 \mathrm{~F}$ & 109.5 \\
\hline $\mathrm{H} 12 \mathrm{D}-\mathrm{C} 12 \mathrm{~B}-\mathrm{H} 12 \mathrm{~F}$ & 109.5 \\
\hline $\mathrm{H} 12 \mathrm{E}-\mathrm{C} 12 \mathrm{~B}-\mathrm{H} 12 \mathrm{~F}$ & 109.5 \\
\hline $\mathrm{C} 14 \mathrm{~B}-\mathrm{C} 13 \mathrm{~B}-\mathrm{C} 18 \mathrm{~B}$ & $119.97(15)$ \\
\hline $\mathrm{C} 14 \mathrm{~B}-\mathrm{C} 13 \mathrm{~B}-\mathrm{C} 4 \mathrm{~B}$ & $118.23(14)$ \\
\hline $\mathrm{C} 18 \mathrm{~B}-\mathrm{C} 13 \mathrm{~B}-\mathrm{C} 4 \mathrm{~B}$ & $121.79(14)$ \\
\hline $\mathrm{C} 15 \mathrm{~B}-\mathrm{C} 14 \mathrm{~B}-\mathrm{C} 13 \mathrm{~B}$ & $121.06(16)$ \\
\hline $\mathrm{C} 15 \mathrm{~B}-\mathrm{C} 14 \mathrm{~B}-\mathrm{H} 14 \mathrm{~B}$ & 119.5 \\
\hline $\mathrm{C} 13 \mathrm{~B}-\mathrm{C} 14 \mathrm{~B}-\mathrm{H} 14 \mathrm{~B}$ & 119.5 \\
\hline $\mathrm{C} 14 \mathrm{~B}-\mathrm{C} 15 \mathrm{~B}-\mathrm{C} 16 \mathrm{~B}$ & $119.40(16)$ \\
\hline $\mathrm{C} 14 \mathrm{~B}-\mathrm{C} 15 \mathrm{~B}-\mathrm{H} 15 \mathrm{~B}$ & 120.3 \\
\hline $\mathrm{C} 16 \mathrm{~B}-\mathrm{C} 15 \mathrm{~B}-\mathrm{H} 15 \mathrm{~B}$ & 120.3 \\
\hline $\mathrm{C} 17 \mathrm{~B}-\mathrm{C} 16 \mathrm{~B}-\mathrm{C} 15 \mathrm{~B}$ & $119.89(16)$ \\
\hline
\end{tabular}




\begin{tabular}{|c|c|c|c|}
\hline $\mathrm{C} 15 \mathrm{~A}-\mathrm{C} 16 \mathrm{~A}-\mathrm{H} 16 \mathrm{~A}$ & 120.0 & $\mathrm{C} 17 \mathrm{~B}-\mathrm{C} 16 \mathrm{~B}-\mathrm{H} 16 \mathrm{~B}$ & 120.1 \\
\hline $\mathrm{C} 17 \mathrm{~A}-\mathrm{C} 16 \mathrm{~A}-\mathrm{H} 16 \mathrm{~A}$ & 120.0 & $\mathrm{C} 15 \mathrm{~B}-\mathrm{C} 16 \mathrm{~B}-\mathrm{H} 16 \mathrm{~B}$ & 120.1 \\
\hline $\mathrm{C} 16 \mathrm{~A}-\mathrm{C} 17 \mathrm{~A}-\mathrm{C} 18 \mathrm{~A}$ & $121.45(16)$ & $\mathrm{C} 16 \mathrm{~B}-\mathrm{C} 17 \mathrm{~B}-\mathrm{C} 18 \mathrm{~B}$ & $121.45(16)$ \\
\hline $\mathrm{C} 16 \mathrm{~A}-\mathrm{C} 17 \mathrm{~A}-\mathrm{H} 17 \mathrm{~A}$ & 119.3 & $\mathrm{C} 16 \mathrm{~B}-\mathrm{C} 17 \mathrm{~B}-\mathrm{H} 17 \mathrm{~B}$ & 119.3 \\
\hline $\mathrm{C} 18 \mathrm{~A}-\mathrm{C} 17 \mathrm{~A}-\mathrm{H} 17 \mathrm{~A}$ & 119.3 & $\mathrm{C} 18 \mathrm{~B}-\mathrm{C} 17 \mathrm{~B}-\mathrm{H} 17 \mathrm{~B}$ & 119.3 \\
\hline $\mathrm{C} 17 \mathrm{~A}-\mathrm{C} 18 \mathrm{~A}-\mathrm{C} 13 \mathrm{~A}$ & $118.21(15)$ & $\mathrm{C} 13 \mathrm{~B}-\mathrm{C} 18 \mathrm{~B}-\mathrm{C} 17 \mathrm{~B}$ & $118.16(15)$ \\
\hline $\mathrm{C} 17 \mathrm{~A}-\mathrm{C} 18 \mathrm{~A}-\mathrm{P} 1 \mathrm{~A}$ & $123.97(13)$ & $\mathrm{C} 13 \mathrm{~B}-\mathrm{C} 18 \mathrm{~B}-\mathrm{P} 1 \mathrm{~B}$ & $117.90(12)$ \\
\hline $\mathrm{C} 13 \mathrm{~A}-\mathrm{C} 18 \mathrm{~A}-\mathrm{P} 1 \mathrm{~A}$ & $117.78(12)$ & $\mathrm{C} 17 \mathrm{~B}-\mathrm{C} 18 \mathrm{~B}-\mathrm{P} 1 \mathrm{~B}$ & $123.72(13)$ \\
\hline $\mathrm{C} 20 \mathrm{~A}-\mathrm{C} 19 \mathrm{~A}-\mathrm{C} 24 \mathrm{~A}$ & $111.30(15)$ & $\mathrm{C} 20 \mathrm{~B}-\mathrm{C} 19 \mathrm{~B}-\mathrm{C} 24 \mathrm{~B}$ & $110.52(15)$ \\
\hline $\mathrm{C} 20 \mathrm{~A}-\mathrm{C} 19 \mathrm{~A}-\mathrm{P} 1 \mathrm{~A}$ & $116.03(12)$ & $\mathrm{C} 20 \mathrm{~B}-\mathrm{C} 19 \mathrm{~B}-\mathrm{P} 1 \mathrm{~B}$ & $116.21(12)$ \\
\hline $\mathrm{C} 24 \mathrm{~A}-\mathrm{C} 19 \mathrm{~A}-\mathrm{P} 1 \mathrm{~A}$ & $112.09(12)$ & $\mathrm{C} 24 \mathrm{~B}-\mathrm{C} 19 \mathrm{~B}-\mathrm{P} 1 \mathrm{~B}$ & $111.95(12)$ \\
\hline $\mathrm{C} 20 \mathrm{~A}-\mathrm{C} 19 \mathrm{~A}-\mathrm{H} 19 \mathrm{~A}$ & 105.5 & $\mathrm{C} 20 \mathrm{~B}-\mathrm{C} 19 \mathrm{~B}-\mathrm{H} 19 \mathrm{~B}$ & 105.8 \\
\hline $\mathrm{C} 24 \mathrm{~A}-\mathrm{C} 19 \mathrm{~A}-\mathrm{H} 19 \mathrm{~A}$ & 105.5 & $\mathrm{C} 24 \mathrm{~B}-\mathrm{C} 19 \mathrm{~B}-\mathrm{H} 19 \mathrm{~B}$ & 105.8 \\
\hline $\mathrm{P} 1 \mathrm{~A}-\mathrm{C} 19 \mathrm{~A}-\mathrm{H} 19 \mathrm{~A}$ & 105.5 & $\mathrm{P} 1 \mathrm{~B}-\mathrm{C} 19 \mathrm{~B}-\mathrm{H} 19 \mathrm{~B}$ & 105.8 \\
\hline $\mathrm{C} 19 \mathrm{~A}-\mathrm{C} 20 \mathrm{~A}-\mathrm{C} 21 \mathrm{~A}$ & $111.30(14)$ & $\mathrm{C} 21 \mathrm{~B}-\mathrm{C} 20 \mathrm{~B}-\mathrm{C} 19 \mathrm{~B}$ & $111.89(15)$ \\
\hline $\mathrm{C} 19 \mathrm{~A}-\mathrm{C} 20 \mathrm{~A}-\mathrm{H} 20 \mathrm{~A}$ & 109.4 & $\mathrm{C} 21 \mathrm{~B}-\mathrm{C} 20 \mathrm{~B}-\mathrm{H} 20 \mathrm{C}$ & 109.2 \\
\hline $\mathrm{C} 21 \mathrm{~A}-\mathrm{C} 20 \mathrm{~A}-\mathrm{H} 20 \mathrm{~A}$ & 109.4 & $\mathrm{C} 19 \mathrm{~B}-\mathrm{C} 20 \mathrm{~B}-\mathrm{H} 20 \mathrm{C}$ & 109.2 \\
\hline $\mathrm{C} 19 \mathrm{~A}-\mathrm{C} 20 \mathrm{~A}-\mathrm{H} 20 \mathrm{~B}$ & 109.4 & $\mathrm{C} 21 \mathrm{~B}-\mathrm{C} 20 \mathrm{~B}-\mathrm{H} 20 \mathrm{D}$ & 109.2 \\
\hline $\mathrm{C} 21 \mathrm{~A}-\mathrm{C} 20 \mathrm{~A}-\mathrm{H} 20 \mathrm{~B}$ & 109.4 & $\mathrm{C} 19 \mathrm{~B}-\mathrm{C} 20 \mathrm{~B}-\mathrm{H} 20 \mathrm{D}$ & 109.2 \\
\hline $\mathrm{H} 20 \mathrm{~A}-\mathrm{C} 20 \mathrm{~A}-\mathrm{H} 20 \mathrm{~B}$ & 108.0 & $\mathrm{H} 20 \mathrm{C}-\mathrm{C} 20 \mathrm{~B}-\mathrm{H} 20 \mathrm{D}$ & 107.9 \\
\hline $\mathrm{C} 22 \mathrm{~A}-\mathrm{C} 21 \mathrm{~A}-\mathrm{C} 20 \mathrm{~A}$ & $110.94(16)$ & $\mathrm{C} 22 \mathrm{~B}-\mathrm{C} 21 \mathrm{~B}-\mathrm{C} 20 \mathrm{~B}$ & $111.02(16)$ \\
\hline $\mathrm{C} 22 \mathrm{~A}-\mathrm{C} 21 \mathrm{~A}-\mathrm{H} 21 \mathrm{~A}$ & 109.5 & $\mathrm{C} 22 \mathrm{~B}-\mathrm{C} 21 \mathrm{~B}-\mathrm{H} 21 \mathrm{C}$ & 109.4 \\
\hline $\mathrm{C} 20 \mathrm{~A}-\mathrm{C} 21 \mathrm{~A}-\mathrm{H} 21 \mathrm{~A}$ & 109.5 & $\mathrm{C} 20 \mathrm{~B}-\mathrm{C} 21 \mathrm{~B}-\mathrm{H} 21 \mathrm{C}$ & 109.4 \\
\hline $\mathrm{C} 22 \mathrm{~A}-\mathrm{C} 21 \mathrm{~A}-\mathrm{H} 21 \mathrm{~B}$ & 109.5 & $\mathrm{C} 22 \mathrm{~B}-\mathrm{C} 21 \mathrm{~B}-\mathrm{H} 21 \mathrm{D}$ & 109.4 \\
\hline $\mathrm{C} 20 \mathrm{~A}-\mathrm{C} 21 \mathrm{~A}-\mathrm{H} 21 \mathrm{~B}$ & 109.5 & $\mathrm{C} 20 \mathrm{~B}-\mathrm{C} 21 \mathrm{~B}-\mathrm{H} 21 \mathrm{D}$ & 109.4 \\
\hline $\mathrm{H} 21 \mathrm{~A}-\mathrm{C} 21 \mathrm{~A}-\mathrm{H} 21 \mathrm{~B}$ & 108.0 & $\mathrm{H} 21 \mathrm{C}-\mathrm{C} 21 \mathrm{~B}-\mathrm{H} 21 \mathrm{D}$ & 108.0 \\
\hline $\mathrm{C} 23 \mathrm{~A}-\mathrm{C} 22 \mathrm{~A}-\mathrm{C} 21 \mathrm{~A}$ & $110.46(16)$ & $\mathrm{C} 23 \mathrm{~B}-\mathrm{C} 22 \mathrm{~B}-\mathrm{C} 21 \mathrm{~B}$ & $110.62(16)$ \\
\hline $\mathrm{C} 23 \mathrm{~A}-\mathrm{C} 22 \mathrm{~A}-\mathrm{H} 22 \mathrm{~A}$ & 109.6 & $\mathrm{C} 23 \mathrm{~B}-\mathrm{C} 22 \mathrm{~B}-\mathrm{H} 22 \mathrm{C}$ & 109.5 \\
\hline $\mathrm{C} 21 \mathrm{~A}-\mathrm{C} 22 \mathrm{~A}-\mathrm{H} 22 \mathrm{~A}$ & 109.6 & $\mathrm{C} 21 \mathrm{~B}-\mathrm{C} 22 \mathrm{~B}-\mathrm{H} 22 \mathrm{C}$ & 109.5 \\
\hline $\mathrm{C} 23 \mathrm{~A}-\mathrm{C} 22 \mathrm{~A}-\mathrm{H} 22 \mathrm{~B}$ & 109.6 & $\mathrm{C} 23 \mathrm{~B}-\mathrm{C} 22 \mathrm{~B}-\mathrm{H} 22 \mathrm{D}$ & 109.5 \\
\hline $\mathrm{C} 21 \mathrm{~A}-\mathrm{C} 22 \mathrm{~A}-\mathrm{H} 22 \mathrm{~B}$ & 109.6 & $\mathrm{C} 21 \mathrm{~B}-\mathrm{C} 22 \mathrm{~B}-\mathrm{H} 22 \mathrm{D}$ & 109.5 \\
\hline $\mathrm{H} 22 \mathrm{~A}-\mathrm{C} 22 \mathrm{~A}-\mathrm{H} 22 \mathrm{~B}$ & 108.1 & $\mathrm{H} 22 \mathrm{C}-\mathrm{C} 22 \mathrm{~B}-\mathrm{H} 22 \mathrm{D}$ & 108.1 \\
\hline $\mathrm{C} 22 \mathrm{~A}-\mathrm{C} 23 \mathrm{~A}-\mathrm{C} 24 \mathrm{~A}$ & $111.86(16)$ & $\mathrm{C} 22 \mathrm{~B}-\mathrm{C} 23 \mathrm{~B}-\mathrm{C} 24 \mathrm{~B}$ & $111.55(15)$ \\
\hline $\mathrm{C} 22 \mathrm{~A}-\mathrm{C} 23 \mathrm{~A}-\mathrm{H} 23 \mathrm{~A}$ & 109.2 & $\mathrm{C} 22 \mathrm{~B}-\mathrm{C} 23 \mathrm{~B}-\mathrm{H} 23 \mathrm{C}$ & 109.3 \\
\hline $\mathrm{C} 24 \mathrm{~A}-\mathrm{C} 23 \mathrm{~A}-\mathrm{H} 23 \mathrm{~A}$ & 109.2 & $\mathrm{C} 24 \mathrm{~B}-\mathrm{C} 23 \mathrm{~B}-\mathrm{H} 23 \mathrm{C}$ & 109.3 \\
\hline $\mathrm{C} 22 \mathrm{~A}-\mathrm{C} 23 \mathrm{~A}-\mathrm{H} 23 \mathrm{~B}$ & 109.2 & $\mathrm{C} 22 \mathrm{~B}-\mathrm{C} 23 \mathrm{~B}-\mathrm{H} 23 \mathrm{D}$ & 109.3 \\
\hline $\mathrm{C} 24 \mathrm{~A}-\mathrm{C} 23 \mathrm{~A}-\mathrm{H} 23 \mathrm{~B}$ & 109.2 & $\mathrm{C} 24 \mathrm{~B}-\mathrm{C} 23 \mathrm{~B}-\mathrm{H} 23 \mathrm{D}$ & 109.3 \\
\hline $\mathrm{H} 23 \mathrm{~A}-\mathrm{C} 23 \mathrm{~A}-\mathrm{H} 23 \mathrm{~B}$ & 107.9 & $\mathrm{H} 23 \mathrm{C}-\mathrm{C} 23 \mathrm{~B}-\mathrm{H} 23 \mathrm{D}$ & 108.0 \\
\hline $\mathrm{C} 19 \mathrm{~A}-\mathrm{C} 24 \mathrm{~A}-\mathrm{C} 23 \mathrm{~A}$ & $111.26(15)$ & $\mathrm{C} 23 \mathrm{~B}-\mathrm{C} 24 \mathrm{~B}-\mathrm{C} 19 \mathrm{~B}$ & $111.15(15)$ \\
\hline $\mathrm{C} 19 \mathrm{~A}-\mathrm{C} 24 \mathrm{~A}-\mathrm{H} 24 \mathrm{~A}$ & 109.4 & $\mathrm{C} 23 \mathrm{~B}-\mathrm{C} 24 \mathrm{~B}-\mathrm{H} 24 \mathrm{C}$ & 109.4 \\
\hline $\mathrm{C} 23 \mathrm{~A}-\mathrm{C} 24 \mathrm{~A}-\mathrm{H} 24 \mathrm{~A}$ & 109.4 & $\mathrm{C} 19 \mathrm{~B}-\mathrm{C} 24 \mathrm{~B}-\mathrm{H} 24 \mathrm{C}$ & 109.4 \\
\hline $\mathrm{C} 19 \mathrm{~A}-\mathrm{C} 24 \mathrm{~A}-\mathrm{H} 24 \mathrm{~B}$ & 109.4 & $\mathrm{C} 23 \mathrm{~B}-\mathrm{C} 24 \mathrm{~B}-\mathrm{H} 24 \mathrm{D}$ & 109.4 \\
\hline $\mathrm{C} 23 \mathrm{~A}-\mathrm{C} 24 \mathrm{~A}-\mathrm{H} 24 \mathrm{~B}$ & 109.4 & $\mathrm{C} 19 \mathrm{~B}-\mathrm{C} 24 \mathrm{~B}-\mathrm{H} 24 \mathrm{D}$ & 109.4 \\
\hline $\mathrm{H} 24 \mathrm{~A}-\mathrm{C} 24 \mathrm{~A}-\mathrm{H} 24 \mathrm{~B}$ & 108.0 & $\mathrm{H} 24 \mathrm{C}-\mathrm{C} 24 \mathrm{~B}-\mathrm{H} 24 \mathrm{D}$ & 108.0 \\
\hline $\mathrm{C} 26 \mathrm{~A}-\mathrm{C} 25 \mathrm{~A}-\mathrm{C} 30 \mathrm{~A}$ & $109.39(14)$ & $\mathrm{C} 30 \mathrm{~B}-\mathrm{C} 25 \mathrm{~B}-\mathrm{C} 26 \mathrm{~B}$ & $109.85(14)$ \\
\hline $\mathrm{C} 26 \mathrm{~A}-\mathrm{C} 25 \mathrm{~A}-\mathrm{P} 1 \mathrm{~A}$ & $107.90(11)$ & $\mathrm{C} 30 \mathrm{~B}-\mathrm{C} 25 \mathrm{~B}-\mathrm{P} 1 \mathrm{~B}$ & $111.27(11)$ \\
\hline $\mathrm{C} 30 \mathrm{~A}-\mathrm{C} 25 \mathrm{~A}-\mathrm{P} 1 \mathrm{~A}$ & $110.88(11)$ & $\mathrm{C} 26 \mathrm{~B}-\mathrm{C} 25 \mathrm{~B}-\mathrm{P} 1 \mathrm{~B}$ & $107.78(11)$ \\
\hline $\mathrm{C} 26 \mathrm{~A}-\mathrm{C} 25 \mathrm{~A}-\mathrm{H} 25 \mathrm{~A}$ & 109.5 & $\mathrm{C} 30 \mathrm{~B}-\mathrm{C} 25 \mathrm{~B}-\mathrm{H} 25 \mathrm{~B}$ & 109.3 \\
\hline
\end{tabular}




\begin{tabular}{|c|c|c|c|}
\hline $\mathrm{C} 30 \mathrm{~A}-\mathrm{C} 25 \mathrm{~A}-\mathrm{H} 25 \mathrm{~A}$ & 109.5 & $\mathrm{C} 26 \mathrm{~B}-\mathrm{C} 25 \mathrm{~B}-\mathrm{H} 25 \mathrm{~B}$ & 109.3 \\
\hline $\mathrm{P} 1 \mathrm{~A}-\mathrm{C} 25 \mathrm{~A}-\mathrm{H} 25 \mathrm{~A}$ & 109.5 & $\mathrm{P} 1 \mathrm{~B}-\mathrm{C} 25 \mathrm{~B}-\mathrm{H} 25 \mathrm{~B}$ & 109.3 \\
\hline $\mathrm{C} 27 \mathrm{~A}-\mathrm{C} 26 \mathrm{~A}-\mathrm{C} 25 \mathrm{~A}$ & $111.96(14)$ & $\mathrm{C} 27 \mathrm{~B}-\mathrm{C} 26 \mathrm{~B}-\mathrm{C} 25 \mathrm{~B}$ & $111.70(14)$ \\
\hline $\mathrm{C} 27 \mathrm{~A}-\mathrm{C} 26 \mathrm{~A}-\mathrm{H} 26 \mathrm{~A}$ & 109.2 & $\mathrm{C} 27 \mathrm{~B}-\mathrm{C} 26 \mathrm{~B}-\mathrm{H} 26 \mathrm{C}$ & 109.3 \\
\hline $\mathrm{C} 25 \mathrm{~A}-\mathrm{C} 26 \mathrm{~A}-\mathrm{H} 26 \mathrm{~A}$ & 109.2 & $\mathrm{C} 25 \mathrm{~B}-\mathrm{C} 26 \mathrm{~B}-\mathrm{H} 26 \mathrm{C}$ & 109.3 \\
\hline $\mathrm{C} 27 \mathrm{~A}-\mathrm{C} 26 \mathrm{~A}-\mathrm{H} 26 \mathrm{~B}$ & 109.2 & $\mathrm{C} 27 \mathrm{~B}-\mathrm{C} 26 \mathrm{~B}-\mathrm{H} 26 \mathrm{D}$ & 109.3 \\
\hline $\mathrm{C} 25 \mathrm{~A}-\mathrm{C} 26 \mathrm{~A}-\mathrm{H} 26 \mathrm{~B}$ & 109.2 & $\mathrm{C} 25 \mathrm{~B}-\mathrm{C} 26 \mathrm{~B}-\mathrm{H} 26 \mathrm{D}$ & 109.3 \\
\hline $\mathrm{H} 26 \mathrm{~A}-\mathrm{C} 26 \mathrm{~A}-\mathrm{H} 26 \mathrm{~B}$ & 107.9 & $\mathrm{H} 26 \mathrm{C}-\mathrm{C} 26 \mathrm{~B}-\mathrm{H} 26 \mathrm{D}$ & 107.9 \\
\hline $\mathrm{C} 26 \mathrm{~A}-\mathrm{C} 27 \mathrm{~A}-\mathrm{C} 28 \mathrm{~A}$ & $110.49(14)$ & $\mathrm{C} 26 \mathrm{~B}-\mathrm{C} 27 \mathrm{~B}-\mathrm{C} 28 \mathrm{~B}$ & $110.84(15)$ \\
\hline $\mathrm{C} 26 \mathrm{~A}-\mathrm{C} 27 \mathrm{~A}-\mathrm{H} 27 \mathrm{~A}$ & 109.6 & $\mathrm{C} 26 \mathrm{~B}-\mathrm{C} 27 \mathrm{~B}-\mathrm{H} 27 \mathrm{C}$ & 109.5 \\
\hline $\mathrm{C} 28 \mathrm{~A}-\mathrm{C} 27 \mathrm{~A}-\mathrm{H} 27 \mathrm{~A}$ & 109.6 & $\mathrm{C} 28 \mathrm{~B}-\mathrm{C} 27 \mathrm{~B}-\mathrm{H} 27 \mathrm{C}$ & 109.5 \\
\hline $\mathrm{C} 26 \mathrm{~A}-\mathrm{C} 27 \mathrm{~A}-\mathrm{H} 27 \mathrm{~B}$ & 109.6 & $\mathrm{C} 26 \mathrm{~B}-\mathrm{C} 27 \mathrm{~B}-\mathrm{H} 27 \mathrm{D}$ & 109.5 \\
\hline $\mathrm{C} 28 \mathrm{~A}-\mathrm{C} 27 \mathrm{~A}-\mathrm{H} 27 \mathrm{~B}$ & 109.6 & $\mathrm{C} 28 \mathrm{~B}-\mathrm{C} 27 \mathrm{~B}-\mathrm{H} 27 \mathrm{D}$ & 109.5 \\
\hline $\mathrm{H} 27 \mathrm{~A}-\mathrm{C} 27 \mathrm{~A}-\mathrm{H} 27 \mathrm{~B}$ & 108.1 & $\mathrm{H} 27 \mathrm{C}-\mathrm{C} 27 \mathrm{~B}-\mathrm{H} 27 \mathrm{D}$ & 108.1 \\
\hline $\mathrm{C} 29 \mathrm{~A}-\mathrm{C} 28 \mathrm{~A}-\mathrm{C} 27 \mathrm{~A}$ & $110.67(15)$ & $\mathrm{C} 29 \mathrm{~B}-\mathrm{C} 28 \mathrm{~B}-\mathrm{C} 27 \mathrm{~B}$ & $110.81(15)$ \\
\hline $\mathrm{C} 29 \mathrm{~A}-\mathrm{C} 28 \mathrm{~A}-\mathrm{H} 28 \mathrm{~A}$ & 109.5 & $\mathrm{C} 29 \mathrm{~B}-\mathrm{C} 28 \mathrm{~B}-\mathrm{H} 28 \mathrm{C}$ & 109.5 \\
\hline $\mathrm{C} 27 \mathrm{~A}-\mathrm{C} 28 \mathrm{~A}-\mathrm{H} 28 \mathrm{~A}$ & 109.5 & $\mathrm{C} 27 \mathrm{~B}-\mathrm{C} 28 \mathrm{~B}-\mathrm{H} 28 \mathrm{C}$ & 109.5 \\
\hline $\mathrm{C} 29 \mathrm{~A}-\mathrm{C} 28 \mathrm{~A}-\mathrm{H} 28 \mathrm{~B}$ & 109.5 & $\mathrm{C} 29 \mathrm{~B}-\mathrm{C} 28 \mathrm{~B}-\mathrm{H} 28 \mathrm{D}$ & 109.5 \\
\hline $\mathrm{C} 27 \mathrm{~A}-\mathrm{C} 28 \mathrm{~A}-\mathrm{H} 28 \mathrm{~B}$ & 109.5 & $\mathrm{C} 27 \mathrm{~B}-\mathrm{C} 28 \mathrm{~B}-\mathrm{H} 28 \mathrm{D}$ & 109.5 \\
\hline $\mathrm{H} 28 \mathrm{~A}-\mathrm{C} 28 \mathrm{~A}-\mathrm{H} 28 \mathrm{~B}$ & 108.1 & $\mathrm{H} 28 \mathrm{C}-\mathrm{C} 28 \mathrm{~B}-\mathrm{H} 28 \mathrm{D}$ & 108.1 \\
\hline $\mathrm{C} 28 \mathrm{~A}-\mathrm{C} 29 \mathrm{~A}-\mathrm{C} 30 \mathrm{~A}$ & $112.09(15)$ & $\mathrm{C} 28 \mathrm{~B}-\mathrm{C} 29 \mathrm{~B}-\mathrm{C} 30 \mathrm{~B}$ & $111.36(15)$ \\
\hline $\mathrm{C} 28 \mathrm{~A}-\mathrm{C} 29 \mathrm{~A}-\mathrm{H} 29 \mathrm{~A}$ & 109.2 & $\mathrm{C} 28 \mathrm{~B}-\mathrm{C} 29 \mathrm{~B}-\mathrm{H} 29 \mathrm{C}$ & 109.4 \\
\hline $\mathrm{C} 30 \mathrm{~A}-\mathrm{C} 29 \mathrm{~A}-\mathrm{H} 29 \mathrm{~A}$ & 109.2 & $\mathrm{C} 30 \mathrm{~B}-\mathrm{C} 29 \mathrm{~B}-\mathrm{H} 29 \mathrm{C}$ & 109.4 \\
\hline $\mathrm{C} 28 \mathrm{~A}-\mathrm{C} 29 \mathrm{~A}-\mathrm{H} 29 \mathrm{~B}$ & 109.2 & $\mathrm{C} 28 \mathrm{~B}-\mathrm{C} 29 \mathrm{~B}-\mathrm{H} 29 \mathrm{D}$ & 109.4 \\
\hline $\mathrm{C} 30 \mathrm{~A}-\mathrm{C} 29 \mathrm{~A}-\mathrm{H} 29 \mathrm{~B}$ & 109.2 & $\mathrm{C} 30 \mathrm{~B}-\mathrm{C} 29 \mathrm{~B}-\mathrm{H} 29 \mathrm{D}$ & 109.4 \\
\hline $\mathrm{H} 29 \mathrm{~A}-\mathrm{C} 29 \mathrm{~A}-\mathrm{H} 29 \mathrm{~B}$ & 107.9 & $\mathrm{H} 29 \mathrm{C}-\mathrm{C} 29 \mathrm{~B}-\mathrm{H} 29 \mathrm{D}$ & 108.0 \\
\hline $\mathrm{C} 29 \mathrm{~A}-\mathrm{C} 30 \mathrm{~A}-\mathrm{C} 25 \mathrm{~A}$ & $111.79(14)$ & $\mathrm{C} 29 \mathrm{~B}-\mathrm{C} 30 \mathrm{~B}-\mathrm{C} 25 \mathrm{~B}$ & $111.57(14)$ \\
\hline $\mathrm{C} 29 \mathrm{~A}-\mathrm{C} 30 \mathrm{~A}-\mathrm{H} 30 \mathrm{~A}$ & 109.3 & $\mathrm{C} 29 \mathrm{~B}-\mathrm{C} 30 \mathrm{~B}-\mathrm{H} 30 \mathrm{C}$ & 109.3 \\
\hline $\mathrm{C} 25 \mathrm{~A}-\mathrm{C} 30 \mathrm{~A}-\mathrm{H} 30 \mathrm{~A}$ & 109.3 & $\mathrm{C} 25 \mathrm{~B}-\mathrm{C} 30 \mathrm{~B}-\mathrm{H} 30 \mathrm{C}$ & 109.3 \\
\hline $\mathrm{C} 29 \mathrm{~A}-\mathrm{C} 30 \mathrm{~A}-\mathrm{H} 30 \mathrm{~B}$ & 109.3 & $\mathrm{C} 29 \mathrm{~B}-\mathrm{C} 30 \mathrm{~B}-\mathrm{H} 30 \mathrm{D}$ & 109.3 \\
\hline $\mathrm{C} 25 \mathrm{~A}-\mathrm{C} 30 \mathrm{~A}-\mathrm{H} 30 \mathrm{~B}$ & 109.3 & $\mathrm{C} 25 \mathrm{~B}-\mathrm{C} 30 \mathrm{~B}-\mathrm{H} 30 \mathrm{D}$ & 109.3 \\
\hline $\mathrm{H} 30 \mathrm{~A}-\mathrm{C} 30 \mathrm{~A}-\mathrm{H} 30 \mathrm{~B}$ & 107.9 & $\mathrm{H} 30 \mathrm{C}-\mathrm{C} 30 \mathrm{~B}-\mathrm{H} 30 \mathrm{D}$ & 108.0 \\
\hline $\mathrm{C} 6 \mathrm{~A}-\mathrm{C} 1 \mathrm{~A}-\mathrm{C} 2 \mathrm{~A}-\mathrm{C} 3 \mathrm{~A}$ & $-0.2(3)$ & $\mathrm{C} 6 \mathrm{~B}-\mathrm{C} 1 \mathrm{~B}-\mathrm{C} 2 \mathrm{~B}-\mathrm{C} 3 \mathrm{~B}$ & $0.4(3)$ \\
\hline $\mathrm{C} 7 \mathrm{~A}-\mathrm{O} 1 \mathrm{~A}-\mathrm{C} 3 \mathrm{~A}-\mathrm{C} 2 \mathrm{~A}$ & $22.6(2)$ & $\mathrm{C} 7 \mathrm{~B}-\mathrm{O} 1 \mathrm{~B}-\mathrm{C} 3 \mathrm{~B}-\mathrm{C} 2 \mathrm{~B}$ & $21.1(2)$ \\
\hline $\mathrm{C} 7 \mathrm{~A}-\mathrm{O} 1 \mathrm{~A}-\mathrm{C} 3 \mathrm{~A}-\mathrm{C} 4 \mathrm{~A}$ & $-159.49(14)$ & $\mathrm{C} 7 \mathrm{~B}-\mathrm{O} 1 \mathrm{~B}-\mathrm{C} 3 \mathrm{~B}-\mathrm{C} 4 \mathrm{~B}$ & $-160.94(14)$ \\
\hline $\mathrm{C} 1 \mathrm{~A}-\mathrm{C} 2 \mathrm{~A}-\mathrm{C} 3 \mathrm{~A}-\mathrm{O} 1 \mathrm{~A}$ & $175.60(16)$ & $\mathrm{C} 1 \mathrm{~B}-\mathrm{C} 2 \mathrm{~B}-\mathrm{C} 3 \mathrm{~B}-\mathrm{O} 1 \mathrm{~B}$ & $176.52(15)$ \\
\hline $\mathrm{C} 1 \mathrm{~A}-\mathrm{C} 2 \mathrm{~A}-\mathrm{C} 3 \mathrm{~A}-\mathrm{C} 4 \mathrm{~A}$ & $-2.2(2)$ & $\mathrm{C} 1 \mathrm{~B}-\mathrm{C} 2 \mathrm{~B}-\mathrm{C} 3 \mathrm{~B}-\mathrm{C} 4 \mathrm{~B}$ & $-1.3(2)$ \\
\hline $\mathrm{O} 1 \mathrm{~A}-\mathrm{C} 3 \mathrm{~A}-\mathrm{C} 4 \mathrm{~A}-\mathrm{C} 5 \mathrm{~A}$ & $-175.61(14)$ & $\mathrm{O} 1 \mathrm{~B}-\mathrm{C} 3 \mathrm{~B}-\mathrm{C} 4 \mathrm{~B}-\mathrm{C} 5 \mathrm{~B}$ & $-177.37(14)$ \\
\hline $\mathrm{C} 2 \mathrm{~A}-\mathrm{C} 3 \mathrm{~A}-\mathrm{C} 4 \mathrm{~A}-\mathrm{C} 5 \mathrm{~A}$ & $2.4(2)$ & $\mathrm{C} 2 \mathrm{~B}-\mathrm{C} 3 \mathrm{~B}-\mathrm{C} 4 \mathrm{~B}-\mathrm{C} 5 \mathrm{~B}$ & $0.7(2)$ \\
\hline $\mathrm{O} 1 \mathrm{~A}-\mathrm{C} 3 \mathrm{~A}-\mathrm{C} 4 \mathrm{~A}-\mathrm{C} 13 \mathrm{~A}$ & $5.6(2)$ & $\mathrm{O} 1 \mathrm{~B}-\mathrm{C} 3 \mathrm{~B}-\mathrm{C} 4 \mathrm{~B}-\mathrm{C} 13 \mathrm{~B}$ & $5.6(2)$ \\
\hline $\mathrm{C} 2 \mathrm{~A}-\mathrm{C} 3 \mathrm{~A}-\mathrm{C} 4 \mathrm{~A}-\mathrm{C} 13 \mathrm{~A}$ & $-176.36(15)$ & $\mathrm{C} 2 \mathrm{~B}-\mathrm{C} 3 \mathrm{~B}-\mathrm{C} 4 \mathrm{~B}-\mathrm{C} 13 \mathrm{~B}$ & $-176.37(15)$ \\
\hline $\mathrm{C} 10 \mathrm{~A}-\mathrm{O} 2 \mathrm{~A}-\mathrm{C} 5 \mathrm{~A}-\mathrm{C} 6 \mathrm{~A}$ & $-2.7(2)$ & $\mathrm{C} 10 \mathrm{~B}-\mathrm{O} 2 \mathrm{~B}-\mathrm{C} 5 \mathrm{~B}-\mathrm{C} 6 \mathrm{~B}$ & $-2.8(2)$ \\
\hline $\mathrm{C} 10 \mathrm{~A}-\mathrm{O} 2 \mathrm{~A}-\mathrm{C} 5 \mathrm{~A}-\mathrm{C} 4 \mathrm{~A}$ & $177.65(14)$ & $\mathrm{C} 10 \mathrm{~B}-\mathrm{O} 2 \mathrm{~B}-\mathrm{C} 5 \mathrm{~B}-\mathrm{C} 4 \mathrm{~B}$ & $176.58(13)$ \\
\hline $\mathrm{C} 3 \mathrm{~A}-\mathrm{C} 4 \mathrm{~A}-\mathrm{C} 5 \mathrm{~A}-\mathrm{O} 2 \mathrm{~A}$ & $179.42(14)$ & $\mathrm{C} 3 \mathrm{~B}-\mathrm{C} 4 \mathrm{~B}-\mathrm{C} 5 \mathrm{~B}-\mathrm{O} 2 \mathrm{~B}$ & $-178.51(14)$ \\
\hline $\mathrm{C} 13 \mathrm{~A}-\mathrm{C} 4 \mathrm{~A}-\mathrm{C} 5 \mathrm{~A}-\mathrm{O} 2 \mathrm{~A}$ & $-1.8(2)$ & $\mathrm{C} 13 \mathrm{~B}-\mathrm{C} 4 \mathrm{~B}-\mathrm{C} 5 \mathrm{~B}-\mathrm{O} 2 \mathrm{~B}$ & $-1.5(2)$ \\
\hline $\mathrm{C} 3 \mathrm{~A}-\mathrm{C} 4 \mathrm{~A}-\mathrm{C} 5 \mathrm{~A}-\mathrm{C} 6 \mathrm{~A}$ & $-0.3(2)$ & $\mathrm{C} 3 \mathrm{~B}-\mathrm{C} 4 \mathrm{~B}-\mathrm{C} 5 \mathrm{~B}-\mathrm{C} 6 \mathrm{~B}$ & $0.9(2)$ \\
\hline $\mathrm{C} 13 \mathrm{~A}-\mathrm{C} 4 \mathrm{~A}-\mathrm{C} 5 \mathrm{~A}-\mathrm{C} 6 \mathrm{~A}$ & $178.46(15)$ & $\mathrm{C} 13 \mathrm{~B}-\mathrm{C} 4 \mathrm{~B}-\mathrm{C} 5 \mathrm{~B}-\mathrm{C} 6 \mathrm{~B}$ & $177.94(15)$ \\
\hline
\end{tabular}




\begin{tabular}{|c|c|c|c|}
\hline $\mathrm{C} 2 \mathrm{~A}-\mathrm{C} 1 \mathrm{~A}-\mathrm{C} 6 \mathrm{~A}-\mathrm{C} 5 \mathrm{~A}$ & $2.2(3)$ & $\mathrm{C} 2 \mathrm{~B}-\mathrm{C} 1 \mathrm{~B}-\mathrm{C} 6 \mathrm{~B}-\mathrm{C} 5 \mathrm{~B}$ & $1.1(3)$ \\
\hline $\mathrm{O} 2 \mathrm{~A}-\mathrm{C} 5 \mathrm{~A}-\mathrm{C} 6 \mathrm{~A}-\mathrm{C} 1 \mathrm{~A}$ & $178.38(16)$ & $\mathrm{O} 2 \mathrm{~B}-\mathrm{C} 5 \mathrm{~B}-\mathrm{C} 6 \mathrm{~B}-\mathrm{C} 1 \mathrm{~B}$ & $177.55(15)$ \\
\hline $\mathrm{C} 4 \mathrm{~A}-\mathrm{C} 5 \mathrm{~A}-\mathrm{C} 6 \mathrm{~A}-\mathrm{C} 1 \mathrm{~A}$ & $-2.0(2)$ & $\mathrm{C} 4 \mathrm{~B}-\mathrm{C} 5 \mathrm{~B}-\mathrm{C} 6 \mathrm{~B}-\mathrm{C} 1 \mathrm{~B}$ & $-1.8(2)$ \\
\hline $\mathrm{C} 3 \mathrm{~A}-\mathrm{O} 1 \mathrm{~A}-\mathrm{C} 7 \mathrm{~A}-\mathrm{C} 9 \mathrm{~A}$ & $67.99(18)$ & $\mathrm{C} 3 \mathrm{~B}-\mathrm{O} 1 \mathrm{~B}-\mathrm{C} 7 \mathrm{~B}-\mathrm{C} 8 \mathrm{~B}$ & $-169.65(14)$ \\
\hline $\mathrm{C} 3 \mathrm{~A}-\mathrm{O} 1 \mathrm{~A}-\mathrm{C} 7 \mathrm{~A}-\mathrm{C} 8 \mathrm{~A}$ & $-170.30(14)$ & $\mathrm{C} 3 \mathrm{~B}-\mathrm{O} 1 \mathrm{~B}-\mathrm{C} 7 \mathrm{~B}-\mathrm{C} 9 \mathrm{~B}$ & $68.52(19)$ \\
\hline $\mathrm{C} 5 \mathrm{~A}-\mathrm{O} 2 \mathrm{~A}-\mathrm{C} 10 \mathrm{~A}-\mathrm{C} 11 \mathrm{~A}$ & $167.36(14)$ & $\mathrm{C} 5 \mathrm{~B}-\mathrm{O} 2 \mathrm{~B}-\mathrm{C} 10 \mathrm{~B}-\mathrm{C} 11 \mathrm{~B}$ & $164.71(14)$ \\
\hline $\mathrm{C} 5 \mathrm{~A}-\mathrm{O} 2 \mathrm{~A}-\mathrm{C} 10 \mathrm{~A}-\mathrm{C} 12 \mathrm{~A}$ & $-72.79(19)$ & $\mathrm{C} 5 \mathrm{~B}-\mathrm{O} 2 \mathrm{~B}-\mathrm{C} 10 \mathrm{~B}-\mathrm{C} 12 \mathrm{~B}$ & $-74.49(18)$ \\
\hline $\mathrm{C} 5 \mathrm{~A}-\mathrm{C} 4 \mathrm{~A}-\mathrm{C} 13 \mathrm{~A}-\mathrm{C} 14 \mathrm{~A}$ & $-96.12(19)$ & $\mathrm{C} 3 \mathrm{~B}-\mathrm{C} 4 \mathrm{~B}-\mathrm{C} 13 \mathrm{~B}-\mathrm{C} 14 \mathrm{~B}$ & $73.2(2)$ \\
\hline $\mathrm{C} 3 \mathrm{~A}-\mathrm{C} 4 \mathrm{~A}-\mathrm{C} 13 \mathrm{~A}-\mathrm{C} 14 \mathrm{~A}$ & $82.6(2)$ & $\mathrm{C} 5 \mathrm{~B}-\mathrm{C} 4 \mathrm{~B}-\mathrm{C} 13 \mathrm{~B}-\mathrm{C} 14 \mathrm{~B}$ & $-103.78(18)$ \\
\hline $\mathrm{C} 5 \mathrm{~A}-\mathrm{C} 4 \mathrm{~A}-\mathrm{C} 13 \mathrm{~A}-\mathrm{C} 18 \mathrm{~A}$ & $83.7(2)$ & $\mathrm{C} 3 \mathrm{~B}-\mathrm{C} 4 \mathrm{~B}-\mathrm{C} 13 \mathrm{~B}-\mathrm{C} 18 \mathrm{~B}$ & $-105.83(19)$ \\
\hline $\mathrm{C} 3 \mathrm{~A}-\mathrm{C} 4 \mathrm{~A}-\mathrm{C} 13 \mathrm{~A}-\mathrm{C} 18 \mathrm{~A}$ & $-97.6(2)$ & $\mathrm{C} 5 \mathrm{~B}-\mathrm{C} 4 \mathrm{~B}-\mathrm{C} 13 \mathrm{~B}-\mathrm{C} 18 \mathrm{~B}$ & $77.2(2)$ \\
\hline $\mathrm{C} 18 \mathrm{~A}-\mathrm{C} 13 \mathrm{~A}-\mathrm{C} 14 \mathrm{~A}-\mathrm{C} 15 \mathrm{~A}$ & $-1.1(3)$ & $\mathrm{C} 18 \mathrm{~B}-\mathrm{C} 13 \mathrm{~B}-\mathrm{C} 14 \mathrm{~B}-\mathrm{C} 15 \mathrm{~B}$ & $-1.8(2)$ \\
\hline $\mathrm{C} 4 \mathrm{~A}-\mathrm{C} 13 \mathrm{~A}-\mathrm{C} 14 \mathrm{~A}-\mathrm{C} 15 \mathrm{~A}$ & $178.67(16)$ & $\mathrm{C} 4 \mathrm{~B}-\mathrm{C} 13 \mathrm{~B}-\mathrm{C} 14 \mathrm{~B}-\mathrm{C} 15 \mathrm{~B}$ & $179.17(15)$ \\
\hline $\mathrm{C} 13 \mathrm{~A}-\mathrm{C} 14 \mathrm{~A}-\mathrm{C} 15 \mathrm{~A}-\mathrm{C} 16 \mathrm{~A}$ & $0.5(3)$ & $\mathrm{C} 13 \mathrm{~B}-\mathrm{C} 14 \mathrm{~B}-\mathrm{C} 15 \mathrm{~B}-\mathrm{C} 16 \mathrm{~B}$ & $-0.3(2)$ \\
\hline $\mathrm{C} 14 \mathrm{~A}-\mathrm{C} 15 \mathrm{~A}-\mathrm{C} 16 \mathrm{~A}-\mathrm{C} 17 \mathrm{~A}$ & $0.1(3)$ & $\mathrm{C} 14 \mathrm{~B}-\mathrm{C} 15 \mathrm{~B}-\mathrm{C} 16 \mathrm{~B}-\mathrm{C} 17 \mathrm{~B}$ & $1.2(2)$ \\
\hline $\mathrm{C} 15 \mathrm{~A}-\mathrm{C} 16 \mathrm{~A}-\mathrm{C} 17 \mathrm{~A}-\mathrm{C} 18 \mathrm{~A}$ & $0.0(3)$ & $\mathrm{C} 15 \mathrm{~B}-\mathrm{C} 16 \mathrm{~B}-\mathrm{C} 17 \mathrm{~B}-\mathrm{C} 18 \mathrm{~B}$ & $0.0(2)$ \\
\hline $\mathrm{C} 16 \mathrm{~A}-\mathrm{C} 17 \mathrm{~A}-\mathrm{C} 18 \mathrm{~A}-\mathrm{C} 13 \mathrm{~A}$ & $-0.7(2)$ & $\mathrm{C} 14 \mathrm{~B}-\mathrm{C} 13 \mathrm{~B}-\mathrm{C} 18 \mathrm{~B}-\mathrm{C} 17 \mathrm{~B}$ & $3.0(2)$ \\
\hline $\mathrm{C} 16 \mathrm{~A}-\mathrm{C} 17 \mathrm{~A}-\mathrm{C} 18 \mathrm{~A}-\mathrm{P} 1 \mathrm{~A}$ & $176.77(13)$ & $\mathrm{C} 4 \mathrm{~B}-\mathrm{C} 13 \mathrm{~B}-\mathrm{C} 18 \mathrm{~B}-\mathrm{C} 17 \mathrm{~B}$ & $-178.03(14)$ \\
\hline $\mathrm{C} 14 \mathrm{~A}-\mathrm{C} 13 \mathrm{~A}-\mathrm{C} 18 \mathrm{~A}-\mathrm{C} 17 \mathrm{~A}$ & $1.2(2)$ & $\mathrm{C} 14 \mathrm{~B}-\mathrm{C} 13 \mathrm{~B}-\mathrm{C} 18 \mathrm{~B}-\mathrm{P} 1 \mathrm{~B}$ & $-171.87(12)$ \\
\hline $\mathrm{C} 4 \mathrm{~A}-\mathrm{C} 13 \mathrm{~A}-\mathrm{C} 18 \mathrm{~A}-\mathrm{C} 17 \mathrm{~A}$ & $-178.58(15)$ & $\mathrm{C} 4 \mathrm{~B}-\mathrm{C} 13 \mathrm{~B}-\mathrm{C} 18 \mathrm{~B}-\mathrm{P} 1 \mathrm{~B}$ & $7.1(2)$ \\
\hline $\mathrm{C} 14 \mathrm{~A}-\mathrm{C} 13 \mathrm{~A}-\mathrm{C} 18 \mathrm{~A}-\mathrm{P} 1 \mathrm{~A}$ & $-176.40(12)$ & $\mathrm{C} 16 \mathrm{~B}-\mathrm{C} 17 \mathrm{~B}-\mathrm{C} 18 \mathrm{~B}-\mathrm{C} 13 \mathrm{~B}$ & $-2.1(2)$ \\
\hline $\mathrm{C} 4 \mathrm{~A}-\mathrm{C} 13 \mathrm{~A}-\mathrm{C} 18 \mathrm{~A}-\mathrm{P} 1 \mathrm{~A}$ & $3.8(2)$ & $\mathrm{C} 16 \mathrm{~B}-\mathrm{C} 17 \mathrm{~B}-\mathrm{C} 18 \mathrm{~B}-\mathrm{P} 1 \mathrm{~B}$ & $172.39(13)$ \\
\hline $\mathrm{C} 25 \mathrm{~A}-\mathrm{P} 1 \mathrm{~A}-\mathrm{C} 18 \mathrm{~A}-\mathrm{C} 17 \mathrm{~A}$ & $29.19(16)$ & $\mathrm{C} 25 \mathrm{~B}-\mathrm{P} 1 \mathrm{~B}-\mathrm{C} 18 \mathrm{~B}-\mathrm{C} 13 \mathrm{~B}$ & $-159.33(13)$ \\
\hline $\mathrm{C} 19 \mathrm{~A}-\mathrm{P} 1 \mathrm{~A}-\mathrm{C} 18 \mathrm{~A}-\mathrm{C} 17 \mathrm{~A}$ & $-79.14(15)$ & $\mathrm{C} 19 \mathrm{~B}-\mathrm{P} 1 \mathrm{~B}-\mathrm{C} 18 \mathrm{~B}-\mathrm{C} 13 \mathrm{~B}$ & $93.19(13)$ \\
\hline $\mathrm{C} 25 \mathrm{~A}-\mathrm{P} 1 \mathrm{~A}-\mathrm{C} 18 \mathrm{~A}-\mathrm{C} 13 \mathrm{~A}$ & $-153.34(13)$ & $\mathrm{C} 25 \mathrm{~B}-\mathrm{P} 1 \mathrm{~B}-\mathrm{C} 18 \mathrm{~B}-\mathrm{C} 17 \mathrm{~B}$ & $26.15(16)$ \\
\hline $\mathrm{C} 19 \mathrm{~A}-\mathrm{P} 1 \mathrm{~A}-\mathrm{C} 18 \mathrm{~A}-\mathrm{C} 13 \mathrm{~A}$ & $98.32(13)$ & $\mathrm{C} 19 \mathrm{~B}-\mathrm{P} 1 \mathrm{~B}-\mathrm{C} 18 \mathrm{~B}-\mathrm{C} 17 \mathrm{~B}$ & $-81.32(15)$ \\
\hline $\mathrm{C} 18 \mathrm{~A}-\mathrm{P} 1 \mathrm{~A}-\mathrm{C} 19 \mathrm{~A}-\mathrm{C} 20 \mathrm{~A}$ & $69.43(14)$ & $\mathrm{C} 18 \mathrm{~B}-\mathrm{P} 1 \mathrm{~B}-\mathrm{C} 19 \mathrm{~B}-\mathrm{C} 20 \mathrm{~B}$ & $72.74(14)$ \\
\hline $\mathrm{C} 25 \mathrm{~A}-\mathrm{P} 1 \mathrm{~A}-\mathrm{C} 19 \mathrm{~A}-\mathrm{C} 20 \mathrm{~A}$ & $-34.95(15)$ & $\mathrm{C} 25 \mathrm{~B}-\mathrm{P} 1 \mathrm{~B}-\mathrm{C} 19 \mathrm{~B}-\mathrm{C} 20 \mathrm{~B}$ & $-31.67(15)$ \\
\hline $\mathrm{C} 18 \mathrm{~A}-\mathrm{P} 1 \mathrm{~A}-\mathrm{C} 19 \mathrm{~A}-\mathrm{C} 24 \mathrm{~A}$ & $-161.19(13)$ & $\mathrm{C} 18 \mathrm{~B}-\mathrm{P} 1 \mathrm{~B}-\mathrm{C} 19 \mathrm{~B}-\mathrm{C} 24 \mathrm{~B}$ & $-158.96(12)$ \\
\hline $\mathrm{C} 25 \mathrm{~A}-\mathrm{P} 1 \mathrm{~A}-\mathrm{C} 19 \mathrm{~A}-\mathrm{C} 24 \mathrm{~A}$ & $94.43(14)$ & $\mathrm{C} 25 \mathrm{~B}-\mathrm{P} 1 \mathrm{~B}-\mathrm{C} 19 \mathrm{~B}-\mathrm{C} 24 \mathrm{~B}$ & $96.63(13)$ \\
\hline $\mathrm{C} 24 \mathrm{~A}-\mathrm{C} 19 \mathrm{~A}-\mathrm{C} 20 \mathrm{~A}-\mathrm{C} 21 \mathrm{~A}$ & $54.5(2)$ & $\mathrm{C} 24 \mathrm{~B}-\mathrm{C} 19 \mathrm{~B}-\mathrm{C} 20 \mathrm{~B}-\mathrm{C} 21 \mathrm{~B}$ & $54.6(2)$ \\
\hline $\mathrm{P} 1 \mathrm{~A}-\mathrm{C} 19 \mathrm{~A}-\mathrm{C} 20 \mathrm{~A}-\mathrm{C} 21 \mathrm{~A}$ & $-175.70(13)$ & $\mathrm{P} 1 \mathrm{~B}-\mathrm{C} 19 \mathrm{~B}-\mathrm{C} 20 \mathrm{~B}-\mathrm{C} 21 \mathrm{~B}$ & $-176.42(13)$ \\
\hline $\mathrm{C} 19 \mathrm{~A}-\mathrm{C} 20 \mathrm{~A}-\mathrm{C} 21 \mathrm{~A}-\mathrm{C} 22 \mathrm{~A}$ & $-56.6(2)$ & $\mathrm{C} 19 \mathrm{~B}-\mathrm{C} 20 \mathrm{~B}-\mathrm{C} 21 \mathrm{~B}-\mathrm{C} 22 \mathrm{~B}$ & $-55.9(2)$ \\
\hline $\mathrm{C} 20 \mathrm{~A}-\mathrm{C} 21 \mathrm{~A}-\mathrm{C} 22 \mathrm{~A}-\mathrm{C} 23 \mathrm{~A}$ & $57.2(2)$ & $\mathrm{C} 20 \mathrm{~B}-\mathrm{C} 21 \mathrm{~B}-\mathrm{C} 22 \mathrm{~B}-\mathrm{C} 23 \mathrm{~B}$ & $56.4(2)$ \\
\hline $\mathrm{C} 21 \mathrm{~A}-\mathrm{C} 22 \mathrm{~A}-\mathrm{C} 23 \mathrm{~A}-\mathrm{C} 24 \mathrm{~A}$ & $-56.6(2)$ & $\mathrm{C} 21 \mathrm{~B}-\mathrm{C} 22 \mathrm{~B}-\mathrm{C} 23 \mathrm{~B}-\mathrm{C} 24 \mathrm{~B}$ & $-56.9(2)$ \\
\hline $\mathrm{C} 20 \mathrm{~A}-\mathrm{C} 19 \mathrm{~A}-\mathrm{C} 24 \mathrm{~A}-\mathrm{C} 23 \mathrm{~A}$ & $-53.4(2)$ & $\mathrm{C} 22 \mathrm{~B}-\mathrm{C} 23 \mathrm{~B}-\mathrm{C} 24 \mathrm{~B}-\mathrm{C} 19 \mathrm{~B}$ & $56.1(2)$ \\
\hline $\mathrm{P} 1 \mathrm{~A}-\mathrm{C} 19 \mathrm{~A}-\mathrm{C} 24 \mathrm{~A}-\mathrm{C} 23 \mathrm{~A}$ & $174.79(13)$ & $\mathrm{C} 20 \mathrm{~B}-\mathrm{C} 19 \mathrm{~B}-\mathrm{C} 24 \mathrm{~B}-\mathrm{C} 23 \mathrm{~B}$ & $-54.35(19)$ \\
\hline $\mathrm{C} 22 \mathrm{~A}-\mathrm{C} 23 \mathrm{~A}-\mathrm{C} 24 \mathrm{~A}-\mathrm{C} 19 \mathrm{~A}$ & $54.8(2)$ & $\mathrm{P} 1 \mathrm{~B}-\mathrm{C} 19 \mathrm{~B}-\mathrm{C} 24 \mathrm{~B}-\mathrm{C} 23 \mathrm{~B}$ & $174.40(12)$ \\
\hline $\mathrm{C} 18 \mathrm{~A}-\mathrm{P} 1 \mathrm{~A}-\mathrm{C} 25 \mathrm{~A}-\mathrm{C} 26 \mathrm{~A}$ & $64.78(12)$ & $\mathrm{C} 18 \mathrm{~B}-\mathrm{P} 1 \mathrm{~B}-\mathrm{C} 25 \mathrm{~B}-\mathrm{C} 30 \mathrm{~B}$ & $-173.24(12)$ \\
\hline $\mathrm{C} 19 \mathrm{~A}-\mathrm{P} 1 \mathrm{~A}-\mathrm{C} 25 \mathrm{~A}-\mathrm{C} 26 \mathrm{~A}$ & $166.96(11)$ & $\mathrm{C} 19 \mathrm{~B}-\mathrm{P} 1 \mathrm{~B}-\mathrm{C} 25 \mathrm{~B}-\mathrm{C} 30 \mathrm{~B}$ & $-72.42(13)$ \\
\hline $\mathrm{C} 18 \mathrm{~A}-\mathrm{P} 1 \mathrm{~A}-\mathrm{C} 25 \mathrm{~A}-\mathrm{C} 30 \mathrm{~A}$ & $-175.44(12)$ & $\mathrm{C} 18 \mathrm{~B}-\mathrm{P} 1 \mathrm{~B}-\mathrm{C} 25 \mathrm{~B}-\mathrm{C} 26 \mathrm{~B}$ & $66.26(13)$ \\
\hline $\mathrm{C} 19 \mathrm{~A}-\mathrm{P} 1 \mathrm{~A}-\mathrm{C} 25 \mathrm{~A}-\mathrm{C} 30 \mathrm{~A}$ & $-73.26(13)$ & $\mathrm{C} 19 \mathrm{~B}-\mathrm{P} 1 \mathrm{~B}-\mathrm{C} 25 \mathrm{~B}-\mathrm{C} 26 \mathrm{~B}$ & $167.08(11)$ \\
\hline $\mathrm{C} 30 \mathrm{~A}-\mathrm{C} 25 \mathrm{~A}-\mathrm{C} 26 \mathrm{~A}-\mathrm{C} 27 \mathrm{~A}$ & $56.37(18)$ & $\mathrm{C} 30 \mathrm{~B}-\mathrm{C} 25 \mathrm{~B}-\mathrm{C} 26 \mathrm{~B}-\mathrm{C} 27 \mathrm{~B}$ & $55.71(19)$ \\
\hline $\mathrm{P} 1 \mathrm{~A}-\mathrm{C} 25 \mathrm{~A}-\mathrm{C} 26 \mathrm{~A}-\mathrm{C} 27 \mathrm{~A}$ & $177.09(11)$ & $\mathrm{P} 1 \mathrm{~B}-\mathrm{C} 25 \mathrm{~B}-\mathrm{C} 26 \mathrm{~B}-\mathrm{C} 27 \mathrm{~B}$ & $177.10(12)$ \\
\hline $\mathrm{C} 25 \mathrm{~A}-\mathrm{C} 26 \mathrm{~A}-\mathrm{C} 27 \mathrm{~A}-\mathrm{C} 28 \mathrm{~A}$ & $-57.73(19)$ & $\mathrm{C} 25 \mathrm{~B}-\mathrm{C} 26 \mathrm{~B}-\mathrm{C} 27 \mathrm{~B}-\mathrm{C} 28 \mathrm{~B}$ & $-56.7(2)$ \\
\hline $\mathrm{C} 26 \mathrm{~A}-\mathrm{C} 27 \mathrm{~A}-\mathrm{C} 28 \mathrm{~A}-\mathrm{C} 29 \mathrm{~A}$ & $56.0(2)$ & $\mathrm{C} 26 \mathrm{~B}-\mathrm{C} 27 \mathrm{~B}-\mathrm{C} 28 \mathrm{~B}-\mathrm{C} 29 \mathrm{~B}$ & $56.3(2)$ \\
\hline $\mathrm{C} 27 \mathrm{~A}-\mathrm{C} 28 \mathrm{~A}-\mathrm{C} 29 \mathrm{~A}-\mathrm{C} 30 \mathrm{~A}$ & $-55.3(2)$ & $\mathrm{C} 27 \mathrm{~B}-\mathrm{C} 28 \mathrm{~B}-\mathrm{C} 29 \mathrm{~B}-\mathrm{C} 30 \mathrm{~B}$ & $-56.1(2)$ \\
\hline $\mathrm{C} 28 \mathrm{~A}-\mathrm{C} 29 \mathrm{~A}-\mathrm{C} 30 \mathrm{~A}-\mathrm{C} 25 \mathrm{~A}$ & $55.2(2)$ & $\mathrm{C} 28 \mathrm{~B}-\mathrm{C} 29 \mathrm{~B}-\mathrm{C} 30 \mathrm{~B}-\mathrm{C} 25 \mathrm{~B}$ & $56.1(2)$ \\
\hline
\end{tabular}


$\mathrm{C} 26 \mathrm{~A}-\mathrm{C} 25 \mathrm{~A}-\mathrm{C} 30 \mathrm{~A}-\mathrm{C} 29 \mathrm{~A} \quad-54.59$ (19)

$\mathrm{P} 1 \mathrm{~A}-\mathrm{C} 25 \mathrm{~A}-\mathrm{C} 30 \mathrm{~A}-\mathrm{C} 29 \mathrm{~A}$
$-173.47(12)$
$\mathrm{C} 26 \mathrm{~B}-\mathrm{C} 25 \mathrm{~B}-\mathrm{C} 30 \mathrm{~B}-\mathrm{C} 29 \mathrm{~B}$

$\mathrm{P} 1 \mathrm{~B}-\mathrm{C} 25 \mathrm{~B}-\mathrm{C} 30 \mathrm{~B}-\mathrm{C} 29 \mathrm{~B}$
$-55.11(19)$

$-174.39(12)$ 